\title{
Treatment of nontuberculous mycobacterial pulmonary disease: an official ATS/ERS/ESCMID/IDSA clinical practice guideline
}

\author{
Charles L. Daley ${ }^{1,2,26}$, Jonathan M. laccarino ${ }^{3}$, Christoph Lange $4,5,6,7,26$, \\ Emmanuelle Cambau ${ }^{8,26}$, Richard J. Wallace Jr ${ }^{9,26}$, Claire Andrejak ${ }^{10,11}$, \\ Erik C. Böttger ${ }^{12}$, Jan Brozek (10) ${ }^{13}$, David E. Griffith ${ }^{14}$, Lorenzo Guglielmetti (1) ${ }^{8,15}$, \\ Gwen A. Huitt (1) ${ }^{1,2}$, Shandra L. Knight ${ }^{16}$, Philip Leitman ${ }^{17}$, Theodore K. Marras ${ }^{18}$ \\ Kenneth N. Olivier (1) ${ }^{19}$, Miguel Santin (1) ${ }^{20}$, Jason E. Stout (1) ${ }^{21}$, Enrico Tortoli (1)22, \\ Jakko van Ingen (1) ${ }^{23}$, Dirk Wagner (10) ${ }^{24}$ and Kevin L. Winthrop ${ }^{25}$
}

@ERSpublications

The official ATS/ERS/ESCMID/IDSA clinical practice guidelines provide 31 evidence-based recommendations for the treatment of nontuberculous mycobacterial (NTM) pulmonary disease https://bit.ly/3fOEwlc

Cite this article as: Daley CL, Iaccarino JM, Lange C, et al. Treatment of nontuberculous mycobacterial pulmonary disease: an official ATS/ERS/ESCMID/IDSA clinical practice guideline. Eur Respir J 2020; 56: 2000535 [https://doi.org/10.1183/13993003.00535-2020].

ABSTRACT Nontuberculous mycobacteria (NTM) represent over 190 species and subspecies, some of which can produce disease in humans of all ages and can affect both pulmonary and extrapulmonary sites. This guideline focuses on pulmonary disease in adults (without cystic fibrosis or human immunodeficiency virus infection) caused by the most common NTM pathogens such as Mycobacterium avium complex, Mycobacterium kansasii, and Mycobacterium xenopi among the slowly growing NTM and Mycobacterium abscessus among the rapidly growing NTM. A panel of experts was carefully selected by leading international respiratory medicine and infectious diseases societies (ATS, ERS, ESCMID, IDSA) and included specialists in pulmonary medicine, infectious diseases and clinical microbiology, laboratory medicine, and patient advocacy. Systematic reviews were conducted around each of 22 PICO (Population, Intervention, Comparator, Outcome) questions and the recommendations were formulated, written, and graded using the GRADE (Grading of Recommendations Assessment, Development, and Evaluation) approach. Thirty-one evidence-based recommendations about treatment of NTM pulmonary disease are provided. This guideline is intended for use by healthcare professionals who care for patients with NTM pulmonary disease, including specialists in infectious diseases and pulmonary diseases.

This article has supplementary material available from erj.ersjournals.com

The guidelines published by the European Respiratory Society (ERS) incorporate data obtained from a comprehensive and systematic literature review of the most recent studies available at the time. Health professionals are encouraged to take the guidelines into account in their clinical practice. However, the recommendations issued by this guideline may not be appropriate for use in all situations. It is the individual responsibility of health professionals to consult other sources of relevant information, to make appropriate and accurate decisions in consideration of each patient's health condition and in consultation with that patient and the patient's caregiver where appropriate and/or necessary, and to verify rules and regulations applicable to drugs and devices at the time of prescription.

Received: 3 March 2020 | Accepted: 3 March 2020

Copyright (C) The Author(s) 2020. This version published by the European Respiratory Society in the European Respiratory Journal, and jointly with Clinical Infectious Diseases. The articles are identical except for minor stylistic and spelling differences in keeping with each journal's style. Either citation can be used when citing this article. 


\section{Executive summary}

The American Thoracic Society (ATS), European Respiratory Society (ERS), European Society of Clinical Microbiology and Infectious Diseases (ESCMID), and Infectious Diseases Society of America (IDSA) jointly sponsored the development of this Guideline to update the treatment recommendations for nontuberculous mycobacterial (NTM) pulmonary disease in adults. NTM represent over 190 species and subspecies (http://www.bacterio.net/mycobacterium.html), many of which can produce disease in humans of all ages and can affect both pulmonary and extrapulmonary sites. Attempting to cover such a broad array of species and disease in a guideline using current guideline development methods is impossible. Therefore, this guideline focuses on pulmonary disease in adults (without cystic fibrosis or human immunodeficiency virus [HIV] infection) caused by the most common NTM pathogens comprising Mycobacterium avium complex (MAC), Mycobacterium kansasii, and Mycobacterium xenopi among the slowly growing NTM and Mycobacterium abscessus among the rapidly growing NTM. Twenty-two PICO (Population, Intervention, Comparators, Outcomes) questions and associated recommendations are included in the Guideline. A panel of experts was carefully selected and screened for conflicts of interest and included specialists in pulmonary medicine, infectious diseases and clinical microbiology, laboratory medicine, and patient advocacy. The recommendations were developed based on the evidence that was appraised using GRADE (Grading of Recommendations Assessment, Development, and Evaluation) and are summarized below $[1,2]$. Recommendations were either "strong" or "conditional" (table 1), and as suggested by GRADE, the phrase "we recommend" was used for strong recommendations and "we suggest” for conditional recommendations [3].

This executive summary is a condensed version of the panel's recommendations for the 22 PICO questions. A detailed description of background, methods, evidence summary, and rationale that support each recommendation can be found online in the full text and accompanying supplementary material.

\section{Diagnostic criteria for NTM pulmonary disease}

The 2007 guideline included clinical, radiographic, and microbiologic criteria for diagnosing NTM pulmonary disease [4]. The current guideline also recommends use of these criteria to classify patients as having NTM pulmonary disease (table 2). The significance of NTM isolated from the sputum of individuals who meet the clinical and radiographic criteria in table 2 must be interpreted in the context of the number of positive cultures and specific species isolated. Because NTM can be isolated from respiratory specimens due to environmental contamination and because some patients who have an NTM isolated from their respiratory tract do not show evidence of progressive disease, $>1$ positive sputum culture is recommended for diagnostic purposes, and the same NTM species (or subspecies in the case of M. abscessus) should be isolated in $\geqslant 2$ sputum cultures. Clinically significant MAC pulmonary disease is

Affiliations: ${ }^{1}$ Dept of Medicine, National Jewish Health, Denver, CO, USA. ${ }^{2}$ Dept of Medicine, University of Colorado School of Medicine, Aurora, CO, USA. ${ }^{3}$ Pulmonary Center, Boston University School of Medicine, Boston, MA, USA. "Division of Clinical Infectious Diseases, Research Center Borstel, Borstel, Germany. ${ }^{5}$ German Center for Infection Research (DZIF), Clinical Tuberculosis Unit, Borstel, Germany. ${ }^{6}$ Respiratory Medicine and International Health, University of Lübeck, Lübeck, Germany. ${ }^{7}$ Dept of Medicine, Karolinska Institute, Stockholm, Sweden. ${ }^{8}$ National Reference Center for Mycobacteria and Antimycobacterial Resistance, APHP -Hôpital Lariboisière, Bacteriology; Inserm, University Paris Diderot, IAME UMR1137, Paris, France. ${ }^{9}$ Mycobacteria/Nocardia Laboratory, Dept of Microbiology, The University of Texas Health Science Center, Tyler, TX, USA. ${ }^{10}$ Respiratory and Intensive Care Unit, University Hospital Amiens, Amiens, France. ${ }^{11}$ EA 4294, AGIR, Jules Verne Picardy University, Amiens, France. ${ }^{12}$ Institute of Medical Microbiology, National Reference Center for Mycobacteria, University of Zurich, Zurich, Switzerland. ${ }^{13}$ Dept of Clinical Epidemiology and Biostatistics, McMaster University Health Sciences Centre, Hamilton, ON, Canada. ${ }^{14}$ Pulmonary Infectious Disease Section, University of Texas Health Science Center, Tyler, TX, USA. ${ }^{15}$ Team E13 (Bactériologie), Centre d'Immunologie et des Maladies Infectieuses, Sorbonne Université, Université Pierre et Marie Curie, Université Paris 06, Centre de Recherche 7, INSERM, IAME UMR1137, Paris, France. ${ }^{16}$ Library and Knowledge Services, National Jewish Health, Denver, CO, USA. ${ }^{17}$ NTM Info and Research, Miami, FL, USA. ${ }^{18}$ Dept of Medicine, University of Toronto and University Health Network, Toronto, ON, Canada. ${ }^{19}$ Pulmonary Branch, National Heart, Lung and Blood Institute, Bethesda, MD, USA. ${ }^{20}$ Service of Infectious Diseases, Bellvitge University Hospital-IDIBELL, University of Barcelona, L'Hospitalet de Llobregat, Barcelona, Spain. ${ }^{21}$ Division of Infectious Diseases and International Health, Duke University Medical Center, Durham, NC, USA. ${ }^{22}$ Emerging Bacterial Pathogens Unit, IRCCS San Raffaele Scientific Institute, Milan, Italy. ${ }^{23}$ Radboud Center for Infectious Diseases, Dept of Medical Microbiology, Radboud University Medical Center, Nijmegen, The Netherlands. ${ }^{24}$ Division of Infectious Diseases, Dept of Medicine II, Medical Center - University of Freiburg, Faculty of Medicine, University of Freiburg, Freiburg, Germany. ${ }^{25}$ Divisions of Infectious Diseases, Schools of Public Health and Medicine, Oregon Health and Science University, Portland, OR, USA. ${ }^{26}$ C.L. Daley, C. Lange, E. Cambau and R.J. Wallace Jr are cochairs of this guideline committee.

Correspondence: Charles L. Daley, National Jewish Health, 1400 Jackson St, Denver, C0 80206, USA. E-mail: daleyclanjhealth.org 
TABLE 1 Interpretation of strong and conditional (weak) recommendations

Recommendations

Conditional

Patients - Most individuals in this situation would want the recommended course of action, and only a small proportion would not.

Clinicians

- Most individuals should receive the intervention.

- Adherence to the recommendation according to the guideline could be used as a quality criterion or performance indicator.

- Formal decision aids are not likely to be needed to help individuals make decisions consistent with their values and preferences.

Policy makers - The recommendation can be adopted as policy in most situations.
- The majority of individuals in this situation would want the suggested course of action, but many would not.

- Recognize that different choices will be appropriate for individual patients and that you must help each patient arrive at a management decision consistent with his or her values and preferences. Decision aids may be useful in helping individuals to make decisions consistent with their values and preferences.

- Policy making will require substantial debate and involvement of various stakeholders.

Source: Grading of Recommendations Assessment, Development and Evaluation Working Group [1, 2].

unlikely in patients who have a single positive sputum culture during the initial evaluation [5-7] but can be as high as $98 \%$ in those with $\geqslant 2$ positive cultures [5]

The pathogenicity of NTM varies significantly from organisms like M. gordonae, which rarely cause disease in humans, to M. kansasii, which should usually be considered pathogenic [8]. For species of low pathogenicity such as M. gordonae, several repeated positive cultures over months, along with strong clinical and radiological evidence of disease, would be required to determine if it was causing disease, whereas a single positive culture for $M$. kansasii in the proper context may be enough evidence to initiate treatment [9]. The pathogenicity of NTM species may differ between geographic areas $[9,10]$.

Importantly, just because a patient meets diagnostic criteria for NTM pulmonary disease does not necessarily mean antibiotic treatment is required. A careful assessment of the pathogenicity of the organism, risks and benefits of therapy, the patient's wish and ability to receive treatment as well as the goals of therapy should be discussed with patients prior to initiating treatment. In some instances, "watchful waiting" may be the preferred course of action.

\section{Recommendations for specific PICO questions}

Twenty-two PICO questions are addressed in this Guideline resulting in 31 recommendations. For each NTM covered, the recommendations are organized by the drugs to be included in the regimen, frequency of administration, and duration of therapy.

TABLE 2 Clinical and microbiologic criteria for diagnosis of nontuberculous mycobacterial pulmonary disease ${ }^{\#}$

Clinical Pulmonary or systemic symptoms

Radiologic Nodular or cavitary opacities on chest radiograph, or a high-resolution computed tomography scan that shows bronchiectasis with multiple small nodules

And Appropriate exclusion of other diagnoses

Microbiologic श 1) Positive culture results from at least two separate expectorated sputum samples. If the results are nondiagnostic, consider repeat sputum AFB smears and cultures or

2) Positive culture results from at least one bronchial wash or lavage

or

3) Transbronchial or other lung biopsy with mycobacterial histologic features (granulomatous inflammation or AFB) and positive culture for NTM or biopsy showing mycobacterial histologic features (granulomatous inflammation or AFB) and one or more sputum or bronchial washings that are culture positive for NTM

Source: Official ATS/IDSA statement [4]. AFB: acid-fast bacilli; NTM: nontuberculous mycobacteria. ${ }^{\#}$ : expert consultation should be obtained when NTM are recovered that are either infrequently encountered or that usually represent environmental contamination. Patients who are suspected of having NTM pulmonary disease but do not meet the diagnostic criteria should be followed until the diagnosis is firmly established or excluded. Making the diagnosis of NTM pulmonary disease does not per se, necessitate the institution of therapy, which is a decision based on the potential risks and benefits of therapy for individual patients. " : when 2 positive cultures are obtained, the isolates should be the same NTM species (or subspecies in the case of $M$. abscessus) in order to meet disease criteria. 
Treatment of NTM pulmonary disease (Questions I-II)

Question I. Should patients with NTM pulmonary disease be treated with antimicrobial therapy or followed for evidence of progression ("watchful waiting")?

\section{Recommendation}

1) In patients who meet the diagnostic criteria for NTM pulmonary disease (table 2), we suggest initiation of treatment rather than watchful waiting, especially in the context of positive acid-fast bacilli sputum smears and/or cavitary lung disease (conditional recommendation, very low certainty in estimates of effect).

Remarks: The decision to initiate antimicrobial therapy for NTM pulmonary disease should be individualized based on a combination of clinical factors, the infecting species, and individual patient priorities. Any treatment decision should include a discussion with the patient that outlines the potential side effects of antimicrobial therapy, the uncertainties surrounding the benefits of antimicrobial therapy, and the potential for recurrence including reinfection (particularly in the setting of nodular/bronchiectatic disease) [11-13].

Question II: Should patients with NTM pulmonary disease be treated empirically or based on in vitro drug susceptibility test results?

\section{Recommendations}

1) In patients with MAC pulmonary disease, we suggest susceptibility-based treatment for macrolides and amikacin over empiric therapy (conditional recommendation, very low certainty in estimates of effect).

2) In patients with $M$. kansasii pulmonary disease, we suggest susceptibility-based treatment for rifampicin over empiric therapy (conditional recommendation, very low certainty in estimates of effect).

3) In patients with $M$. xenopi pulmonary disease, the panel members felt there is insufficient evidence to make a recommendation for or against susceptibility-based treatment.

4) In patients with M. abscessus pulmonary disease we suggest susceptibility-based treatment for macrolides and amikacin over empiric therapy (conditional recommendation, very low certainty in estimates of effect). For macrolides, a 14-day incubation and/or sequencing of the erm(41) gene is required in order to evaluate for potential inducible macrolide resistance.

Remark: Although in vitro-in vivo correlations have not yet been proven for all major antimycobacterial drugs, baseline susceptibility testing to specific drugs is recommended according to the Clinical and Laboratory Standards Institute (CLSI) guidelines $[14,15]$ for NTM isolates from patients with definite disease. Testing of other drugs may be useful, but there is insufficient data to make specific recommendations.

Mycobacterium avium complex (Questions III-IX)

Question III: Should patients with macrolide-susceptible MAC pulmonary disease be treated with a 3-drug regimen with a macrolide or without a macrolide?

\section{Recommendation}

1) In patients with macrolide-susceptible MAC pulmonary disease, we recommend a 3-drug regimen that includes a macrolide over a 3-drug regimen without a macrolide (strong recommendation, very low certainty in estimates of effect).

Remarks: Although no well-designed randomized trials of macrolide therapy have been performed, macrolide susceptibility has been a consistent predictor of treatment success for pulmonary MAC [16-18]. Loss of the macrolide from the treatment regimen is associated with a markedly reduced rate of conversion of sputum cultures to negative and higher mortality [16-18]. Therefore, the panel members felt strongly that a macrolide should be included in the regimen.

Question IV: In patients with newly diagnosed macrolide-susceptible MAC pulmonary disease, should an azithromycin-based regimen or a clarithromycin-based regimen be used?

\section{Recommendation}

1) In patients with macrolide-susceptible MAC pulmonary disease we suggest azithromycin-based treatment regimens rather than clarithromycin-based regimens (conditional recommendation, very low certainty in estimates of effect). 
Remarks: The panel felt that azithromycin was preferred over clarithromycin because of better tolerance, less drug interactions, lower pill burden, single daily dosing, and equal efficacy. However, when azithromycin is not available or not tolerated, clarithromycin is an acceptable alternative.

Question V: Should patients with MAC pulmonary disease be treated with a parenteral amikacin or streptomycin-containing regimen or without a parenteral amikacin or streptomycin-containing regimen?

\section{Recommendation}

1) For patients with cavitary or advanced/severe bronchiectatic or macrolide-resistant MAC pulmonary disease, we suggest that parenteral amikacin or streptomycin be included in the initial treatment regimen (conditional recommendation, moderate certainty in estimates of effect).

Remarks: In the absence of comparably effective oral medications there are few options other than parenteral aminoglycosides for "intensifying" standard oral MAC therapy. The committee thought that the benefits outweighed risks in those patients with cavitary or advanced/severe bronchiectatic or macrolide-resistant MAC pulmonary disease and that administration of at least 2-3 months of an aminoglycoside was the best balance between risks and benefits.

Question VI: In patients with macrolide-susceptible MAC pulmonary disease, should a regimen with inhaled amikacin or a regimen without inhaled amikacin be used for treatment?

\section{Recommendations}

1) In patients with newly diagnosed MAC pulmonary disease, we suggest neither inhaled amikacin (parenteral formulation) nor amikacin liposome inhalation suspension (ALIS) be used as part of the initial treatment regimen (conditional recommendation, very low certainty in estimates of effect).

2) In patients with MAC pulmonary disease who have failed therapy after at least 6 months of guideline-based therapy, we recommend addition of ALIS to the treatment regimen rather than a standard oral regimen, only (strong recommendation, moderate certainty in estimates of effect).

Remarks: Randomized controlled trials have demonstrated the efficacy and safety of ALIS when added to guideline-based therapy for treatment refractory MAC pulmonary disease [19, 20]. ALIS is currently approved by the United States Federal Drug Administration for treatment of refractory MAC pulmonary disease. As noted in question 5, we suggest that parenteral amikacin or streptomycin be included in the initial treatment regimen in patients with cavitary or advanced/severe bronchiectatic or macrolide-resistant MAC pulmonary disease.

Question VII: In patients with macrolide-susceptible MAC pulmonary disease, should a 3-drug or a 2-drug macrolide-containing regimen be used for treatment?

\section{Recommendation}

1) In patients with macrolide-susceptible MAC pulmonary disease, we suggest a treatment regimen with at least 3 drugs (including a macrolide and ethambutol) over a regimen with 2 drugs (a macrolide and ethambutol alone) (conditional recommendation, very low certainty in estimates of effect).

Remarks: A priority in MAC pulmonary disease therapy is preventing the development of macrolide resistance. The panel members were concerned that the currently available data [21] were insufficient to determine the risk of acquired macrolide resistance with a 2-drug regimen and therefore suggest a 3 drug macrolide-containing regimen.

Question VIII: In patients with macrolide susceptible MAC pulmonary disease, should a daily or a 3-times weekly macrolide-based regimen be used for treatment?

\section{Recommendations}

1) In patients with noncavitary nodular/bronchiectatic macrolide-susceptible MAC pulmonary disease, we suggest a 3 times per week macrolide-based regimen rather than a daily macrolide-based regimen (conditional recommendation, very low certainty in estimates of effect).

2) In patients with cavitary or severe/advanced nondular bronchiectatic macrolide-susceptible MAC pulmonary disease we suggest a daily macrolide-based regimen rather than 3 times per week macrolide-based regimen (conditional recommendation, very low certainty in estimates of effect).

Remarks: Intermittent therapy has similar sputum conversion rates as daily therapy for nodular/ bronchiectatic MAC pulmonary disease and is also better tolerated than daily therapy [22, 23]. A critically important finding from the available studies is the lack of development of macrolide resistance with intermittent therapy. There is not similar evidence to justify or support intermittent therapy for cavitary MAC pulmonary disease and it is not recommended. 
Question IX: In patients with macrolide-susceptible MAC pulmonary disease, should patients be treated with $<12$ months of treatment after culture negativity or $\geqslant 12$ months of treatment after culture negativity?

\section{Recommendation}

1) We suggest that patients with macrolide-susceptible MAC pulmonary disease receive treatment for at least 12 months after culture conversion (conditional recommendation, very low certainty in estimates of effect).

Remarks: The optimal duration of therapy for pulmonary MAC disease is not currently known. The panel felt that in the absence of evidence identifying an optimal treatment duration that the recommendation from the 2007 Guideline should be followed [4].

Mycobacterium kansasii (Questions X-XIV)

Question X: In patients with rifampcin-susceptible $M$. kansasii pulmonary disease, should an isoniazid-containing regimen or a macrolide-containing regimen be used for treatment?

\section{Recommendation}

1) In patients with rifampicin-susceptible $M$. kansasii pulmonary disease, we suggest a regimen of rifampicin, ethambutol, and either isoniazid or macrolide (conditional recommendation, very low certainty in estimates of effect).

Remarks: Isoniazid is widely used at present for treatment of $M$. kansasii pulmonary disease, and in the experience of the panel members, there have been good outcomes when using a regimen consisting of rifampicin, ethambutol, and isoniazid irrespective of the result of minimal inhibitory concentrations (MICs) for isoniazid and ethambutol [24]. Based on the in vitro activity of macrolides against M. kansasii, and 2 studies that demonstrated good treatment outcomes when clarithromycin was substituted for isoniazid $[25,26]$, the panel suggests that either isoniazid or a macrolide can be used in combination with rifampicin and ethambutol.

Question XI: In patients with rifampicin-susceptible M. kansasii pulmonary disease, should parenteral amikacin or streptomycin be included in the treatment regimen?

\section{Recommendation}

1) We suggest that neither parenteral amikacin nor streptomycin be used routinely for treating patients with M. kansasii pulmonary disease (strong recommendation, very low certainty in estimates of effect).

Remarks: Regimens of 3 oral agents, rifampicin and ethambutol, and either isoniazid or a macrolide, achieve high rates of sustained culture conversion and treatment success in the treatment of M. kansasii pulmonary disease. Therefore, given the good outcomes observed with oral regimens and the high risk of adverse effects associated with parenteral amikacin or streptomycin, the committee felt strongly that the use of these parenteral agents is not warranted, unless it is impossible to use a rifampicin-based regimen or severe disease is present.

Question XII: In patients with rifampicin-susceptible M. kansasii pulmonary disease, should a treatment regimen that includes a fluoroquinolone or a regimen without a fluoroquinolone be used?

\section{Recommendations}

1) In patients with rifampicin-susceptible M. kansasii pulmonary disease, we suggest using a regimen of rifampicin, ethambutol, and either isoniazid or macrolide instead of a fluoroquinolone (conditional recommendation, very low certainty in estimates of effect).

2) In patients with rifampicin-resistant M. kansasii or intolerance to one of the first-line antibiotics we suggest a fluoroquinolone (e.g. moxifloxacin) be used as part of a second-line regimen (conditional recommendation, very low certainty in estimates of effect).

Remarks: Treatment success of $M$. kansasii pulmonary disease with a rifamycin-based drug regimen is usually excellent but the optimal choice of companion drugs is not clear. While ethambutol is usually the preferred companion drug, the choice of an additional companion drug may be isoniazid, a macrolide or a fluoroquinolone. As there is more experience and better evidence for treatment regimens that include isoniazid or a macrolide as a companion drug, these drugs are preferred [25-28]. For rifampicin-resistant disease, a regimen such as ethambutol, azithromycin, and a fluoroquinolone would be likely to lead to successful treatment.

Question XIII: In patients with rifampicin-susceptible $M$. kansasii pulmonary disease, should a 3 times per week or daily treatment regimen be used? 


\section{Recommendations}

1) In patients with noncavitary nodular/bronchiectatic $M$. kansasii pulmonary disease treated with a rifampicin, ethambutol, and macrolide regimen, we suggest either daily or 3 times weekly treatment (conditional recommendation, very low certainty in estimates of effect)

2) In patients with cavitary M. kansasii pulmonary disease treated with a rifampicin, ethambutol, and macrolide-based regimen, we suggest daily treatment instead of 3 times weekly treatment (conditional recommendation, very low certainty in estimates of effect).

3) In all patients with $M$. kansasii pulmonary disease treated with an isoniazid, ethambutol, and rifampicin regimen, we suggest treatment be given daily instead of 3 times weekly (conditional recommendation, very low certainty in estimates of effect).

Remarks: Because there are no randomized trials available and the small size of the single study that evaluated 3 times weekly therapy [26], the committee did not feel that they could recommend intermittent therapy in the setting of cavitary disease until more evidence was available. Similarly, there are no data to support the use of isoniazid on a 3 times weekly basis in patients with $M$. kansasii pulmonary disease.

Question XIV: In patients with rifampicin susceptible $M$. kansasii pulmonary disease, should treatment be continued for $<12$ months or $\geqslant 12$ months?

\section{Recommendation}

1) We suggest that patients with rifampin susceptible $M$. kansasii pulmonary disease be treated for at least 12 months (conditional recommendation, very low certainty in estimates of effect).

Remarks: Current rifampicin-based treatment regimens are associated with a high rate of success if used for at least 12 months [27, 29]. Randomized controlled trials comparing shorter treatment regimens are currently lacking. Although some experts would favour 12 months of treatment after culture conversion, there is no evidence that relapses could be prevented with treatment courses longer than 12 months. Therefore, the panel members felt that $M$. kansasii could be treated for a fixed duration of 12 months instead of 12 months beyond culture conversion. Because sputum conversion at 4 months of rifampicin-based regimens is usually observed [29-31], expert consultation should be obtained if cultures fail to convert to negative by that time.

Mycobacterium xenopi (Questions XV-XVIII)

Question XV: In patients with $M$. xenopi pulmonary disease, should a treatment regimen that includes a fluoroquinolone or a regimen without a fluoroquinolone be used?

\section{Recommendation}

1) In patients with M. xenopi pulmonary disease, we suggest using a multidrug treatment regimen that includes moxifloxacin or macrolide (conditional recommendation, low certainty in estimates of effect).

Remarks: There is in vitro evidence that macrolides and fluoroquinolones are active against $M$. xenopi, whereas rifampicin and ethambutol are inactive in vitro alone and in combinations [32]. Preliminary data from a study in France that randomized patients to receive either moxifloxacin or clarithromycin plus ethambutol and rifampicin reported no difference in the treatment success between the study arms [33].

Question XVI: In patients with M. xenopi pulmonary disease, should a 2-, 3-, or 4-drug regimen be used for treatment?

\section{Recommendation}

1) In patients with $M$. xenopi pulmonary disease, we suggest a daily regimen that includes at least 3 drugs: rifampicin, ethambutol, and either a macrolide and/or a fluoroquinolone (e.g. moxifloxacin) (conditional recommendation, very low certainty in estimates of effect).

Remarks: Given the high mortality associated with M. xenopi disease, the panel members felt the large risk of treatment failure with a 2-drug regimen warranted at least a 3-drug treatment regimen. However, the absence of universal access to moxifloxacin and the small amount of data for other fluoroquinolones has to be considered when choosing a regimen.

Question XVII: In patients with $M$. xenopi pulmonary disease, should parenteral amikacin or streptomycin be included in the treatment regimen? 


\section{Recommendation}

1) In patients with cavitary or advanced/severe bronchiectatic $M$. xenopi pulmonary disease, we suggest adding parenteral amikacin to the treatment regimen and obtaining expert consultation (conditional recommendation, very low certainty in estimates of effect).

Remarks: Barring compelling evidence to the contrary, M. xenopi patients should be treated aggressively given the high mortality of the disease [34-36]. In addition to the high mortality, the committee considered the general acceptability and feasibility of parenteral therapy, and potential costs and toxicities, all based on clinical experience.

Question XVIII: In patients with M. xenopi pulmonary disease, should treatment be continued for $<12$ months or $\geqslant 12$ months after culture conversion?

\section{Recommendation}

1) In patients with $M$. xenopi pulmonary disease, we suggest that treatment be continued for at least 12 months beyond culture conversion (conditional recommendation, very low certainty in estimates of effect).

Remarks: Data suggest that treatment outcomes improve if the duration of treatment increases [35, 37]. The panel felt that this outweighs the risk of adverse events associated with longer treatment and agrees with previous recommendations [4].

\section{Mycobacterium abscessus (Questions XIX-XXI)}

Question XIX: In patients with $M$. abscessus pulmonary disease, should a macrolide-based regimen or a regimen without a macrolide be used for treatment?

\section{Recommendations}

1) In patients with M. abscessus pulmonary disease caused by strains without inducible or mutational resistance, we recommend a macrolide-containing multidrug treatment regimen (strong recommendation, very low certainty in estimates of effect).

2) In patients with M. abscessus pulmonary disease caused by strains with inducible or mutational macrolide resistance, we suggest a macrolide-containing regimen if the drug is being used for its immunomodulatory properties although the macrolide is not counted as an active drug in the multidrug regimen (conditional recommendation, very low certainty in estimates of effect).

Remarks: M. abscessus infections can be life-threatening, and the use of macrolides is potentially of great benefit. Macrolides are very active in vitro against $M$. abscessus strains without a functional erm(41) gene, and evidence supports use of macrolides in patients with disease caused by macrolide-susceptible $M$. abscessus $[38,39]$. It is important to perform in vitro macrolide susceptibility testing including detection of a functional or nonfunctional erm(41) gene [40-42].

Question XX: In patients with M. abscessus complex pulmonary disease, how many antibiotics should be included within multidrug regimens?

\section{Recommendation}

1) In patients with $M$. abscessus pulmonary disease, we suggest a multidrug regimen that includes at least 3 active drugs (guided by in vitro susceptibility) in the initial phase of treatment (conditional recommendation, very low certainty in estimates of effect).

Remarks: Given the usual disease severity of M. abscessus pulmonary disease, the variable and limited in vitro drug susceptibility of these organisms, the potential for the emergence of drug resistance, and the potential for more rapid progression of $M$. abscessus pulmonary disease, the panel members suggest using a regimen consisting of three or more active drugs. The panel members felt strongly that treatment regimens should be designed in collaboration with experts in the management of these complicated infections.

Question XXI: In patients with M. abscessus pulmonary disease, should shorter or longer duration therapy be used for treatment?

\section{Recommendation}

1) In patients with $M$. abscessus pulmonary disease, we suggest that either a shorter or longer treatment regimen be used and expert consultation obtained (conditional recommendation for either the intervention or the comparison, very low certainty in estimates of effect). 
Remarks: The lack of studies, the variation in drug availability, resources, and practice settings made it difficult to come to a consensus on the optimum duration of therapy. In addition, the panel members felt that some subgroups of patients should be considered separately in determining the length of therapy such as: patients with nodular/bronchiectatic versus cavitary disease, patients affected by lung disease caused by different M. abscessus subspecies and importantly, depending on susceptibility to macrolides and amikacin. The panel members suggest that an expert in the management of patients with M. abscessus pulmonary disease be consulted.

\section{Surgical resection (Question XXII)}

Question XXII: Should surgery plus medical therapy or medical therapy alone be used to treat NTM pulmonary disease?

\section{Recommendation}

1) In selected patients with NTM pulmonary disease, we suggest surgical resection as an adjuvant to medical therapy after expert consultation (conditional recommendation, very low certainty in estimates of effect).

Remarks: Selected patients with failure of medical management, cavitary disease, drug resistant isolates, or complications such as hemoptysis or severe bronchiectasis may undergo surgical resection of the diseased lung. The decision to proceed with surgical resection must be weighed against the risks and benefits of surgery. The panel suggests that surgery be performed by a surgeon experienced in mycobacterial surgery [43].

\section{Background}

The genus Mycobacterium consists of a diverse group of species and subspecies (http://www.bacterio.net/ mycobacterium.html). With the exception of Mycobacterium tuberculosis complex, Mycobacterium leprae complex, and Mycobacterium ulcerans the rest of the species are referred to as NTM, and they can be found throughout our environment. The most common clinical presentation is that of pulmonary disease, often occurring in the setting of underlying structural airway disease such as bronchiectasis or chronic obstructive pulmonary disease [4]. The incidence and prevalence of NTM pulmonary disease are increasing in many areas of the world with rates particularly high in older individuals and those with underlying bronchiectasis [44-48]. The reasons for the increases in prevalence are not fully understood but are likely multifactorial including environmental, host, and microbial factors. Regardless of the reasons for the increase, it is clear that healthcare providers will be encountering these patients increasingly frequently in the coming years.

The availability of gene sequencing has improved taxonomy of mycobacteria, with an extraordinary increase in the number of validly published NTM species. Of the many known NTM species, only a small number appear to cause pulmonary disease in humans. The most common slowly growing NTM to do so are members of Mycobacterium avium complex which now consists of 12 separate species [49]. The most common to cause pulmonary disease are $M$. avium, $M$. intracellulare, and $M$. chimaera. Other important NTM causing pulmonary disease are M. kansasii and M. xenopi. M. abscessus and its subspecies abscessus, bolletii, and massiliense are by far the most common causative agents of pulmonary disease due to rapidly growing mycobacteria.

Diagnosis of NTM pulmonary disease requires the synthesis of clinical, radiographic, and microbiology data. The ATS and IDSA developed a set of criteria to help guide clinicians in determining which patients are likely to have progressive disease [4]. Unfortunately, the predictive values of these criteria are not well studied, and thus they serve primarily as a guide to clinicians. The laboratory remains a critical component in the diagnosis of NTM pulmonary disease given the many species and variable pathogenicity. Identification of NTM to the species level and in the case of M. abscessus, to the subspecies level, can provide important clinical and epidemiologic information.

Treatment of NTM pulmonary disease varies depending on the species (in some cases subspecies), extent of disease, drug susceptibility results (with limitations), and underlying comorbidities. Regimens require the use of multiple antimicrobial agents that are often associated with clinically significant adverse reactions and must be administered for prolonged periods. Even so, treatment outcomes are often suboptimal, and reinfection with another strain or species is common. In many settings, expert consultation is helpful.

\section{Methods}

Committee composition

This guideline was developed by a multidisciplinary committee consisting of physicians and researchers with recognized NTM expertise (C. Andrejak, E.C. Böttger, E. Cambau, C.L. Daley, D.E. Griffith, 
L. Guglielmetti, G.A. Huitt, Jakko van Ingen, C. Lange, T.K. Marras, K.N. Olivier, J.E. Stout, M. Santin, E. Tortoli, D. Wagner, K.L. Winthrop, R.J. Wallace Jr), methodologists (J. Brozek and J.M. Iaccarino), and a representative from an NTM nonprofit organization the goal of which is patient support, education, and research in NTM (P. Leitman). The patient representative was a full participant in each step of the development process but did not vote on specific recommendations. The committee was chaired by C.L. Daley (ATS) and cochaired by C. Lange (ERS), E. Cambau (ESCMID), and R.J. Wallace Jr (IDSA), representing their respective societies. The committee worked with a medical librarian (S.L. Knight) who had expertise in evidence synthesis and the guideline development process. All of the members who had potential financial and/or intellectual conflicts recused themselves or were excused by the chairs from discussions related to the recommendation formulation and grading, and voting on recommendations related to the potential conflict. The methodology team conducted systematic reviews and prepared evidence summaries following the GRADE approach $[1,2]$.

\section{Formulating clinical questions}

The committee developed potential questions to be addressed in the guideline using the 2007 guideline document [4] and their own clinical experience and expertise. Committee members were asked to rank questions in order of importance and priority with all questions deemed important and high priority included for the guideline. Twenty-two questions were chosen based on committee ranking pertinent to the treatment of NTM pulmonary disease. Some of these questions had been previously addressed in 2007 but required updating based on new evidence, whereas others were new questions that the committee felt were critical topics for NTM management. Outcomes of interest were selected a priori by the panel based on their experience and clinical expertise, using the approach suggested by the GRADE working group [1, $2,50]$.

\section{Literature search and review of evidence}

A medical librarian (S.L. Knight) designed a search strategy using medical subject heading keywords and text words (see online supplement) limited to human studies and articles with English abstracts. Databases searched included MEDLINE, EMBASE, Cochrane Registry of Controlled Trials, Health Technology Assessment, and the Database of Abstracts of Reviews of Effects from 1946 through July 2015. An update was performed in May 2016 prior to the final meeting at the ATS International Conference and a final update was performed in June 2018 prior to manuscript submission.

\section{Development of clinical recommendations}

The committee developed recommendations that considered the certainty of the evidence from the GRADE evidence profiles, as well as other domains that inform decision-making. The GRADE evidence-to-decision framework was used to organize and document discussion for each recommendation $[2,50]$. The committee considered each of the following in recommendation development: the quality of the evidence, the balance of desirable and undesirable consequences of compared management options, the values and preferences associated with the decision, the implications for resource use and health equity, the acceptability of the intervention to stakeholders, and the feasibility of implementation (see online supplement). The committee developed recommendations based on the GRADE evidence profiles for each question, with recommendations and their strength decided by committee consensus during face-to-face meetings.

Recommendations were either "strong" or "conditional," according to the GRADE approach (table 1) [3]. Strength of the recommendations was based upon the confidence in the estimates of effect, the outcomes studied and associated importance to patients, the desirable and undesirable consequences of treatment, the cost of treatment, the implications of treatment on health equity, the feasibility of treatment, and the acceptability of treatment to important stakeholders. In instances where there was low certainty in the estimates of effect, the committee determined whether a strong recommendation was warranted based on paradigmatic situations outlined by ANDREws et al. [3]. As suggested by GRADE, the phrase "we recommend" was used for strong recommendations and "we suggest" for conditional recommendations [3]. The guideline, which was funded by ATS, ERS, ESCMID, and IDSA, will be re-evaluated in 4 years to determine if an update is necessary.

\section{Diagnostic criteria for NTM pulmonary disease}

The 2007 guideline included clinical, radiographic and microbiologic criteria for diagnosing NTM pulmonary disease [4]. The current guideline also recommends use of these criteria to classify patients as having NTM pulmonary disease (table 2). The significance of NTM isolated from the sputum of individuals who meet the clinical and radiographic criteria in table 2 must be interpreted in the context of the number of positive cultures and specific species isolated. Because NTM can be isolated from 
respiratory specimens due to environmental contamination and because some patients who have an NTM isolated from their respiratory tract do not show evidence of progressive disease, $>1$ positive sputum culture is recommended for diagnostic purposes and the same NTM species (or subspecies in the case of M. abscessus) should be isolated in $\geqslant 2$ sputum cultures collected over an interval of a week or more. Clinically significant MAC pulmonary disease is unlikely in patients who have a single positive sputum culture during the initial evaluation [5-7] but can be as high as $98 \%$ in those with $\geqslant 2$ positive cultures [5].

The pathogenicity of NTM varies significantly from organisms like $M$. gordonae, which rarely cause disease in humans, to M. kansasii, which should usually be considered pathogenic [8]. For species of low pathogenicity such as M. gordonae, several repeated positive cultures over months, along with strong clinical and radiological evidence of disease, would be required to determine if it was causing disease whereas a single positive culture for $M$. kansasii in the proper context may be enough evidence to initiate treatment [9]. The pathogenicity of NTM species may differ between geographic areas $[9,10]$.

Importantly, just because a patient meets diagnostic criteria for NTM pulmonary disease does not necessarily mean antibiotic treatment is required. A careful assessment of the pathogenicity of the organism, patient's symptoms, risks and benefits of therapy, the patient's wish and ability to receive treatment as well as the goals of therapy should be discussed with patients prior to initiating treatment. In some instances, "watchful waiting" may be the preferred course of action.

\section{Laboratory diagnosis of nontuberculous mycobacterial pulmonary disease}

The clinical laboratory plays a critical role in the diagnosis of NTM pulmonary disease. A detailed review of the subject is beyond the scope of the guideline but a brief review of clinically relevant laboratory issues is below.

\section{Obtaining respiratory samples}

Given the slow course of NTM pulmonary disease, a prolonged interval ensures that repeat positive cultures are unlikely to reflect a transient contamination of the tracheobronchial system after a single environmental exposure. To distinguish NTM pulmonary disease from occasional presence of NTM in the tracheobronchial tract, at least 3 respiratory samples are investigated, over an interval of at least a week. For cavitary NTM pulmonary disease, sputum samples often suffice for diagnosis [4]. Bronchoalveolar lavage fluid and bronchial washing cultures have been reported in several small studies to be more sensitive than spontaneously expectorated sputum culture to diagnose nodular/bronchiectatic NTM disease [51-54]. However, in the largest study, the yield of sputum culture and bronchial washing culture were equivalent [55]. Bronchoscopy is performed only in patients suspected of having NTM pulmonary disease from whom sputum specimens cannot be obtained spontaneously or through induction.

\section{Sample processing and culture}

Decontamination by $0.25 \% \mathrm{~N}$-acetyl-L-cysteine and $1 \% \mathrm{NaOH}$ (NALC-NaOH) is the preferred method. An increase of $\mathrm{NaOH}$ concentrations lowers contamination rates but decreases sensitivity of culture [56].

Culture of respiratory samples is performed on both liquid and solid media, to improve sensitivity. A meta-analysis [57] of 9 studies [58-65] showed an increase in the sensitivity of culture for NTM of $15 \%$ if a solid medium was incubated alongside a liquid culture system. In the few studies that applied multiple solid media and reported results per medium, the Löwenstein-Jensen medium was found to be most sensitive for the detection of NTM $[59,64]$. However, the Clinical and Laboratory Standards Institute (CLSI) currently recommends use of $7 \mathrm{H} 10$ and $7 \mathrm{H} 11$ solid media [66]. CLSI has suggested incubations temperatures of $36 \pm 1^{\circ} \mathrm{C}$ for slow growers and $28 \pm 2^{\circ} \mathrm{C}$ for rapid growers [66]: higher temperatures (i.e. $42^{\circ} \mathrm{C}$ ) might accelerate growth of $M$. xenopi but lower incubation temperatures have not proven useful in diagnosing NTM pulmonary disease [67].

In patients with a high suspicion of NTM pulmonary disease but negative cultures, review of decontamination procedures and use of supplemented media and molecular detection may be helpful although supplemental media are rarely necessary to diagnose NTM pulmonary disease. For molecular detection, most use a Mycobacterium genus specific assay used in conjunction with nucleic acid sequencing, to distinguish M. tuberculosis complex from NTM [68, 69].

\section{Species identification}

Correct identification of NTM is important, as it can predict the clinical relevance of an isolate [8] as well as aid in the selection of a treatment regimen. Both molecular and mass spectrometry-based methods can be applied. Molecular identification is the preferred method and can be achieved using probes or gene sequencing. Probe-based assays are easier to perform and implement but lack discriminatory power, leading to misidentification and an oversimplified view of NTM phylogeny and epidemiology [70, 71]. 
Gene sequencing allows a higher level of discrimination, often up to subspecies level but is only feasible for laboratories with access to sequencing facilities. Several target genes have been described, e.g. 16S rRNA, $h s p 65, r p o B$, and the 16S-23S internal transcribed spacer (ITS) [72-75]. 16S rRNA gene sequencing alone offers limited discriminatory power, particularly for the $M$. abscessus- $M$. chelonae group [70]. The $h s p 65$ and $r p o B$ genes and ITS are more discriminative [76]. Complementing 16S rRNA sequencing with additional targets where required yields the best discriminatory power, allowing identifications up to subspecies level (e.g. for M. abscessus) [77, 78].

The discriminatory power of the matrix-assisted laser desorption ionization-time of flight (MALDI-TOF) mass spectrometry method for NTM has increased with recent improvements in protein extraction protocols and databases but not all species and subspecies can be differentiated with this approach [79, 80]. These procedures work well for pure cultures [80, 81]; however, if applied to newly positive liquid cultures, only $50 \%$ of isolates can be immediately identified [82]. For the remainder, subculture on solid media until the occurrence of visual growth is needed to obtain good MALDI-TOF results [79].

All clinically relevant isolates of NTM should be identified by molecular methods, including follow-up isolates of patients undergoing NTM pulmonary disease treatment. Where possible, isolates from patients who are being treated for NTM pulmonary disease are frozen and saved in order to distinguish reinfection from relapse when recurrence occurs.

\section{Drug susceptibility testing}

In general, drug susceptibility testing is performed for drugs used in treatment regimens and for which there are clear correlations between in vitro activity and the in vivo outcomes of treatment. Such correlations have become increasingly clear for NTM, especially for macrolides and amikacin. CLSI provides guidelines for test procedures [14, 15].

For M. avium complex, there is a clear correlation between baseline macrolide susceptibility of the causative strain and the outcome of treatment with macrolide-ethambutol-rifampicin regimens [83, 84]. Acquired macrolide resistance in $M$. avium complex is due to point mutations in the $23 \mathrm{~S}$ rRNA ( $r r l$ ) gene $[85,86]$. For amikacin, acquired resistance is due to resistance conferring mutations in the $16 \mathrm{~S}$ rRNA (rrs) gene and are mostly isolated from patients with extensive exposure to amikacin and/or related aminoglycosides $[55,87]$. The breakpoint for resistance is a $\mathrm{MIC} \geqslant 64 \mu \mathrm{g} \cdot \mathrm{mL}^{-1}$ for parenteral amikacin and $\geqslant 128 \mu \mathrm{g} \cdot \mathrm{mL}^{-1}$ for amikacin liposome inhalation suspension (ALIS) [15], and finding such MICs would lead to cessation of intravenous or nebulized amikacin therapy [20]. Tentative breakpoints for linezolid and moxifloxacin are also provided by CLSI but for these, in vitro-in vivo correlations have not been established [15].

For M. kansasii, rifampicin and clarithromycin are the key drugs to test. Rifampicin resistance (MIC $>2 \mu \mathrm{g} \cdot \mathrm{mL}^{-1}$ ) is rare but can occur in isolates from patients with significant rifamycin exposures and failure of treatment with a rifamycin containing regimen [15]. Resistance to clarithromycin is defined as an MIC $\geqslant 32 \mu \mathrm{g} \cdot \mathrm{mL}^{-1}[15]$. When rifampicin resistance has been identified, susceptibilities to amikacin, ciprofloxacin, doxycycline, linezolid, minocycline, moxifloxacin, rifabutin, and trimethoprimsulfamethoxazole are tested [88].

In $M$. abscessus pulmonary disease the association between in vitro drug susceptibility and in vivo outcome of treatment is evident for macrolides and amikacin [39, 89, 90]. Parenteral drugs with in vitro activity include amikacin, imipenem, cefoxitin, and tigecycline. Oral drugs with some activity are the macrolides, oxazolidinones (linezolid) and clofazimine. Clofazimine shows in vitro activity, acts synergistically with amikacin and macrolides [91, 92], and prevents the emergence of amikacin-resistant M. abscessus in vitro [92].

Strains of M. abscessus subsp. abscessus and M. abscessus subsp. bolletii have an erythromycin resistance methylase ( $\mathrm{rm})$ gene, named erm(41), that results in inducible resistance to macrolides [93]. This inducible resistance can be measured in vitro by prolonged (i.e. up to 14 days) incubation of microdilution trays [40, 93] or can be investigated by molecular detection and characterization of the erm(41) gene. In M. abscessus subsp massiliense, the erm(41) gene is nonfunctional owing to a large deletion, thus rendering the strains macrolide susceptible. A nonfunctional gene also occurs in some M. abscessus subsp abscessus as a result of a $\mathrm{C}$ instead of a $\mathrm{T}$ at the nucleotide 28 position (Arg10 instead of Trp10) in the erm(41) gene [40, 94]. All of the $3 \mathrm{M}$. abscessus subspecies can develop constitutive macrolide resistance owing to 23S RNA ( $r r l$ ) gene mutations [94]. Susceptibility testing panels for M. abscessus include at least amikacin, cefoxitin, imipenem, clarithromycin, linezolid, doxycycline, tigecycline, ciprofloxacin, and moxifloxacin.

CLSI recommends that drug susceptibility testing be performed by broth microdilution [88]. For patients whose NTM isolate is deemed to be clinically significant, drug susceptibility testing is performed for primary isolates as well as relapse/failure isolates. 


\section{Recommendations for specific PICO questions}

Twenty-two PICO questions are addressed in this guideline. For additional details please see the online supplement, which includes supporting supplemental evidence profiles for each question (tables E3.1-22) and evidence to decision tables (tables E4.1-22) for each recommendation. For specific pathogens ( $M$. avium complex, M. kansasii, M. xenopi, and M. abscessus), the PICO questions are organized by the drugs to be included in the regimen, frequency of administration, and duration of therapy.

\section{Treatment of NTM pulmonary disease (Questions I-II)}

Question I. Should patients with NTM pulmonary disease be treated with antimicrobial therapy or followed for evidence of progression ("watchful waiting")?

Background: Treatment of NTM pulmonary disease with antimicrobial agents offers the possibility of cure of the disease. However, the potential benefits of antimicrobial treatment must be weighed against the potential adverse effects of treatment, low cure rates for some forms of infection, uncertain effect of treatment on quality and quantity of life, high costs of treatment, and the potential for reinfection.

\section{Recommendation}

1) In patients who meet the diagnostic criteria for NTM pulmonary disease (table 2), we suggest initiation of treatment rather than watchful waiting, especially in the context of positive acid-fast bacilli sputum smears and/or cavitary lung disease (conditional recommendation, very low certainty in estimates of effect).

Summary of the evidence: No randomized, controlled trials have been conducted to examine the impact of treatment on either survival or quality of life. Limited retrospective observational data have failed to demonstrate that treatment of NTM pulmonary disease prolongs survival over watchful waiting $[95,96]$. The relative and absolute effect estimates and 95\% confidence intervals (CIs) for each outcome (table E3. 1) and discussion of value preferences, feasibility, cost, acceptability, and health inequality (table E4.1) can be found in the supplement.

Not all patients who have NTM isolated from a respiratory specimen or who meet ATS/IDSA diagnostic criteria will develop progressive NTM pulmonary disease. For example, among 488 patients with MAC pulmonary disease in Taiwan who met ATS/IDSA disease criteria and were followed for at least 1 year, 305 (62.5\%) demonstrated progression of disease [97]. Progression was more likely to occur in patients who were acid-fast bacilli smear positive, had fibrocavitary disease or more extensive radiographic disease. Among those patients who met the 2007 ATS/IDSA criteria for MAC pulmonary disease and in whom treatment was not initiated, 51.6\% underwent spontaneous sputum conversion during a median follow-up of 5.6 years [97]. Predictors of spontaneous sputum culture conversion included younger age, higher body mass index, and negative sputum acid-fast bacilli smears at initial diagnosis.

Observational cohorts have noted wide variability in the proportion of patients with NTM pulmonary disease who are offered treatment (20-81\%) likely contributing to selection bias [95, 98-105]. NTM pulmonary disease has been associated with diminished quality of life that correlates with the severity of lung impairment [106, 107]. A single study using standardized methods for quality of life assessment demonstrated improvement of quality of life associated with treatment of M. abscessus infection [108].

Justification and implementation considerations: The decision to initiate antimicrobial therapy for NTM pulmonary disease should be individualized based on a combination of clinical factors, the infecting species, and individual patient priorities. Factors associated with relatively poor prognosis (e.g. cavitary disease, low body mass index, low albumin, and/or elevated inflammatory markers) [97, 99, 102, 104, 109], isolation of an organism that is more virulent and/or more responsive to antimicrobial therapy (e. g. M. kansasii), and underlying immune suppression were felt to move the balance toward antimicrobial treatment. Major symptoms such as severe fatigue with marked decrease in quality of life can also be major factors in starting therapy. Conversely, mild signs and symptoms of disease, higher potential for medication intolerance/toxicity and organisms less responsive to treatment (e.g. M. abscessus) were felt to move the balance toward watchful waiting. Any treatment decision should include a discussion with the patient that outlines the potential adverse effects of antimicrobial therapy, the uncertainties surrounding the benefits of antimicrobial therapy, and the potential for recurrence including reinfection (particularly in the setting of nodular-bronchiectatic disease) [11-13].

Question II. Should patients with NTM pulmonary disease be treated empirically or based on in vitro drug susceptibility test results?

Background: Drug susceptibility testing for NTM is useful but only for antibiotics for which correlations between in vitro activity and microbiological response to treatment have been well documented $[110,111]$. 
These include the macrolides (clarithromycin and azithromycin) [112] and amikacin [19, 20, 87] with MAC and M. abscessus [19, 113], and rifampicin with M. kansasii [114, 115].

\section{Recommendations}

1) In patients with MAC pulmonary disease, we suggest susceptibility-based treatment for macrolides and amikacin over empiric therapy (conditional recommendation, very low certainty in estimates of effect).

2) In patients with $M$. kansasii pulmonary disease, we suggest susceptibility-based treatment for rifampicin over empiric therapy (conditional recommendation, very low certainty in estimates of effect).

3) In patients with $M$. xenopi pulmonary disease, the committee members feel there is insufficient evidence to make a recommendation for or against susceptibility-based treatment.

4) In patients with $M$. abscessus pulmonary disease we suggest susceptibility-based treatment for macrolides and amikacin over empiric therapy (conditional recommendation, very low certainty in estimates of effect). For macrolides, a 14-day incubation and/or sequencing of the erm(41) gene should be performed to evaluate for potential inducible macrolide resistance.

Summary of the evidence: Only one study was identified that reported treatment outcomes based on empiric treatment versus the results of drug susceptibility results [101]. The study was a retrospective observational study of 31 patients with various species causing NTM pulmonary disease who met the 1997 ATS case definition. Patients were treated with a variety of treatment regimens (13 different combinations were used). Adjusting treatment according to the results of drug susceptibility tests was not associated with any difference in median survival (75\% with adjustment and $80 \%$ without). However, the study suffers from serious methodological flaws including lack of randomization, use of the 1997 ATS diagnostic criteria, and methods of determining and interpreting drug susceptibility that are no longer recommended. Discussion of value preferences, feasibility, cost, acceptability, and health inequality (table E4.2) can be found in the supplement.

Although only 1 study was identified that attempted to evaluate the outcomes of treatment based on drug susceptibility results there are other studies that have correlated outcomes with in vitro activity. Trials of monotherapy with clarithromycin, rifampicin, ethambutol, or clofazimine for HIV-associated disseminated MAC demonstrated that only clarithromycin susceptibility results correlated with treatment outcomes $[113,116]$. In MAC pulmonary disease, retrospective case series $[83,84,112,117,118]$ have also shown that in vitro resistance to clarithromycin was associated with worse outcomes than susceptibility to clarithromycin, and a randomized trial found no association between in vitro susceptibility to either rifampicin or ethambutol and failure/relapse [119]. However, the latter study applied a drug susceptibility method not recommended for NTM and presented and analysed only aggregate resistance data for all groups (MAC, M. xenopi, and M. malmoense) utilizing uniform discrete thresholds rather than considering MICs as a continuous variable to be explored for an association across species.

Amikacin is an important drug used for treatment of $M$. abscessus pulmonary disease. Resistance to amikacin is caused by a specific mutation (A1408G) in the 16S rRNA ( rrs) gene that has been associated with a high MIC $\left(>64 \mu \mathrm{g} \cdot \mathrm{mL}^{-1}\right)$ and previous exposure to amikacin $[87,120]$.

Recent phase II and III clinical trials evaluating the efficacy and safety of ALIS in patients with refractory pulmonary disease due to MAC (or M. abscessus) reported that when there was an A1408G mutation in the $16 \mathrm{~S} \mathrm{rRNA}$ gene and/or the MIC was $>64 \mu \mathrm{g} \cdot \mathrm{mL}^{-1}$ in MAC isolates, no patients achieved culture conversion on ALIS; responses were seen with MIC values up to and including $64 \mu \mathrm{g} \cdot \mathrm{mL}^{-1}[19,20]$. Treatment failure occurred in 2 patients whose isolates had become resistant by mutation to amikacin [19]. In a randomized trial comparing intravenous streptomycin with placebo added to a standard 3-drug regimen, there was no association of treatment outcome with MIC to streptomycin; however, exact MIC values were not determined if above $4 \mu \mathrm{g} \cdot \mathrm{mL}^{-1}[121]$.

For M. kansasii pulmonary disease, resistance to rifampicin has been associated with treatment failure $[114,115]$, although no randomized trials have been conducted that associate baseline MICs to clinical outcome. For $M$. xenopi lung disease, few studies have correlated in vitro activity of specific antimycobacterial drugs with treatment outcomes [36, 101, 122, 123]. No association could be found between in vitro activity and treatment failure/relapse in a randomized trial comparing rifampicin plus ethambutol with or without isoniazid. The study had important limitations including a small sample size and the use of discrete thresholds (based on M. tuberculosis) rather than considering MIC values as a continuous variable [36].

Recent studies have reported poor treatment outcomes associated with macrolide resistance due to either mutational or inducible resistance related to the presence of a functional erm(41) gene in $M$. abscessus 
subsp. abscessus and bolletii. In a retrospective cohort treated with a standard regimen, the presence of in vitro resistance to clarithromycin was associated with worse outcomes [39]. In a follow-up study, patients with $M$. abscessus subsp. massiliense were more likely to convert cultures to negative compared with patients infected with $M$. abscessus subsp. abscessus ( $85 \%$ versus $25 \%, \mathrm{p}<0.001$ ), presumably because of the presence of a nonfunctional erm(41) gene in the former (gene with major deletions) and inducible macrolide resistance due to a functional erm(41) gene in the latter [38, 40-42]. In addition, culture conversion rates were significantly higher in patients infected with an $M$. abscessus subsp. abscessus C28 sequevar isolate that does not exhibit inducible resistance to macrolides [12]. Alternatively, when M. abscessus subsp. massiliense develops mutational macrolide resistance with a mutation in the 23S rRNA gene, culture conversion is similar to that seen with subsp. abscessus and functional $\operatorname{erm}(41)$ gene $[40,124,125]$.

Justification and implementation considerations: Although in vitro-in vivo correlations have been proven only for macrolides, amikacin and rifampicin (the latter only for M. kansasii), baseline susceptibility testing is recommended by CLSI guidelines for NTM isolates from patients with definite disease [14, 15]. Based on studies reviewed above, there is evidence of poor outcomes in cases of macrolide-resistant MAC $[16,112]$ and $M$. abscessus $[38,39]$ and poor outcomes in rifampicin-resistant M. kansasii $[114,115]$. Similarly, recent data from randomized clinical trials evaluating ALIS have demonstrated that high MICs of amikacin are associated with poor microbiological response as reported in a previous retrospective analysis of patients treated with parenteral amikacin [19, 20, 87]. Based on the studies and recommendations above, laboratories should provide drug susceptibility test results for the macrolides and amikacin for MAC and M. abscessus and rifampicin for M. kansasii. Precise subspeciation is helpful for $M$. abscessus as identification of subsp. massiliense is associated with a nonfunctionalerm(41) gene and in vitro susceptibility (MIC below $4 \mu \mathrm{g} \cdot \mathrm{mL}^{-1}$ ) [42], and thus the macrolides are active if constitutive resistance is not present. Alternatively, sequence analysis of the erm(41) gene can provide information (e.g., truncated or C28 sequevar) that can exclude inducible macrolide resistance. Although other drugs are sometimes tested in order to guide $M$. abscessus therapy, there are insufficient data to make specific recommendations in this regard.

Because no studies could be identified that adequately addressed $M$. xenopi pulmonary disease and in the absence of drug susceptibility testing guidelines and breakpoints for M. xenopi, the panel was unable to provide recommendation for or against susceptibility-based treatment.

\section{Treatment of MAC pulmonary disease (Questions III-IX) \\ Question III. Should patients with macrolide-susceptible MAC pulmonary disease be treated with a 3-drug regimen with a macrolide or without a macrolide?}

Background: Macrolides (clarithromycin and azithromycin) have been the basis of therapy against MAC pulmonary disease because they were demonstrated in multiple trials to be effective in prophylaxis and multidrug treatment of disseminated MAC infection [126-130].

\section{Recommendation}

1) In patients with macrolide-susceptible MAC pulmonary disease, we recommend a 3-drug regimen that includes a macrolide over a 3-drug regimen without a macrolide (strong recommendation, very low certainty in estimates of effect).

Summary of the evidence: In spite of the widespread use of macrolides for treating MAC disease, there have been only two randomized controlled trials comparing a macrolide-containing regimen with a nonmacrolide-containing regimen $[131,132]$. A British Thoracic Society trial randomized 170 patients with primarily cavitary MAC pulmonary disease to receive standard doses of rifampicin and ethambutol with either clarithromycin or ciprofloxacin [131]. The results showed that the clarithromycin group had a lower failure/relapse rate than the ciprofloxacin group (13\% versus $23 \%)$ and was tolerated better. However, all-cause mortality was higher in the clarithromycin group for unclear reasons (48\% versus $30 \%$ ). At 5 years only $30 \%$ of the clarithromycin group and $21 \%$ of the ciprofloxacin group were known to have completed therapy and been alive.

In a second small prospective trial from Japan [132], 27 patients with MAC pulmonary disease were treated for 1 year with rifampicin and ethambutol plus either gatifloxacin or low dose $(600 \mathrm{mg})$ clarithromycin. The treatment outcomes were not significantly different between study arms: 11/13 $(84.6 \%)$ in the gatifloxacin group and $9 / 14(64 \%)$ patients in the clarithromycin group achieved sputum culture conversion to negative. The relative and absolute effect estimates and 95\% CIs for each outcome (table E3.3) and discussion of value preferences, feasibility, cost, acceptability, and health inequality (table E4.3) can be found in the supplement. 
The committee was concerned about several aspects of these 2 studies including, a) small sample size, b) underdosing of the macrolide, c) populations not representative of nodular bronchiectatic MAC pulmonary disease patients encountered frequently in clinical practice, d) the use of gatifloxacin which is not approved for use or no longer marketed in many countries worldwide, and e) the high overall mortality seen in one study [131], which raised questions about the validity of the study.

There have been other noncomparator trials of macrolide-containing regimens that have reported varying culture conversion rates. A recent systematic review reported a sustained sputum culture conversion incidence rate ratio of 0.54 (95\% CI $0.45-0.63)$ for macrolide-containing regimens versus $0.38(0.25-0.52)$ for macrolide-free regimens [133]. Sputum conversion increased in the macrolide-containing regimens compared with macrolide-free regimens as study quality improved. Another systematic review reported overall treatment success using macrolide-containing regimens was 52.3\% (95\% CI 44.7\%-59.9\%) and success increased to $61.4 \%$ if treated with an ATS/IDSA 3-drug regimen, and to $65.7 \%$ if further treated for at least 12 months [134]. The companion drugs and length of treatment are important factors in treatment success. Only regimens using rifamycin and ethambutol or clofazimine and ethambutol have been shown to prevent the emergence of macrolide resistance during treatment $[22,135]$.

Perhaps the strongest available evidence for the importance of the macrolide in the treatment regimen is demonstrated by its loss from the regimen. In the setting of macrolide-resistant disease, the sputum culture conversion rate falls from approximately $80 \%[22,23]$ to only $5-36 \%[16-18,136]$.

Justification and implementation considerations: Case series have demonstrated that macrolide-containing regimens are associated with higher culture conversion rates than nonmacrolide-containing regimens [137]. Macrolide susceptibility has been a consistent predictor of treatment success for MAC pulmonary disease, whereas susceptibility to most other drugs has not been a predictor [112]. In a postmarketing study from Japan, among 271 patients with macrolide-susceptible MAC pulmonary disease who received a clarithromycin-based regimen, sputum culture conversion to negative occurred in 95\% [136]. Although no well-designed randomized trials of macrolide therapy have been performed, the panel felt that macrolides are a critical component of MAC treatment based on poor patient outcomes if macrolides are not included in the treatment regimen. As such the panel members voted unanimously to make a strong recommendation despite the very low certainty of estimates of effect.

Question IV. In patients with newly diagnosed macrolide-susceptible MAC pulmonary disease, should an azithromycin-based regimen or a clarithromycin-based regimen be used?

Background: The macrolides are considered to be key components in treatment regimens against MAC pulmonary disease. The 2007 guideline expressed a preference for azithromycin over clarithromycin in initial treatment regimens [4].

\section{Recommendation}

1) In patients with macrolide-susceptible MAC pulmonary disease we suggest azithromycin-based treatment regimens rather than clarithromycin-based regimens (conditional recommendation, very low certainty in estimates of effect).

Summary of the evidence: Both clarithromycin and azithromycin have demonstrated activity in MAC pulmonary disease, with early studies demonstrating some efficacy for monotherapy [117, 138], and subsequent studies demonstrating efficacy as part of multi-drug regimens administered both daily [83] and 3 times weekly [22, 139, 140]. Limited data are available from comparisons of treatment outcomes in patients treated with clarithromycin versus azithromycin [22, 141], and no significant difference was found in either microbiologic efficacy or tolerability, although there was a nonsignificant trend toward lower tolerability for clarithromycin in 1 study [141]. The relative and absolute effect estimates and 95\% CIs for each outcome (table E3.4) and discussion of value preferences, feasibility, cost, acceptability, and health inequality (table E4.4) can be found in the supplement.

A recent systematic review reported no clinically significant differences between azithromycin and clarithromycin in sputum culture conversion at 6 months, end of therapy, or on sustained conversion after treatment nor was there a difference in the acquisition of macrolide resistance [133]. However, azithromycin has less potential for drug-drug interactions than clarithromycin [142]. The drug-drug interactions are particularly relevant when a rifamycin (rifampicin or rifabutin) is given concurrently; azithromycin serum concentrations are affected less by concurrent rifampicin or rifabutin administration than clarithromycin, but the interaction is bidirectional for clarithromycin and rifabutin, leading to increased concentration of rifabutin (but not rifampicin), which has been associated with uveitis [111, 143-145]. Other considerations that would favour azithromycin over clarithromycin include a lower pill burden, once daily dosing, and possibly lower costs. 
Justification and implementation considerations: The preference for azithromycin is primarily based on the expert panel's perception of better tolerability of azithromycin and fewer drug-drug interactions mediated by the cytochrome P450 system [146] than with clarithromycin. Both azithromycin and clarithromycin have been reported to be associated with severe adverse effects, including sudden death presumably mediated by QTc prolongation [147, 148]. However, a systematic review that evaluated adverse events in people taking macrolides versus placebo for any indication reported no increase in cardiac disorders or mortality when compared with placebo [149]. Electrocardiographic monitoring may be considered for patients when concurrent medications that prolong the QTc interval are being used. In the same systematic review noted above [149], hearing loss was reported more frequently in patients taking macrolides than placebo; however, the differences were not statistically significant, and there were no studies of clarithromycin to address differences between macrolides. In older patients, hearing loss and gastrointestinal symptoms have been associated with higher doses (600 mg daily) and serum concentrations of azithromycin [150], whereas bitter taste, nausea, and elevated hepatic enzymes have been associated with higher doses (1000 mg twice daily) of clarithromycin [151]. Of note, all studies included some patients who did not tolerate azithromycin and were successfully switched to clarithromycin and vice versa. Switching from one agent to the other is a strategy that may be considered in case of intolerance. The panel felt that azithromycin was preferred over clarithromycin because of likely better tolerance, less drug interactions, lower pill burden, single daily dosing, and equal efficacy. In places where azithromycin is not available, clarithromycin is an acceptable alternative although more drug interactions are possible.

Question V. Should patients with MAC pulmonary disease be treated with a parenteral amikacin or streptomycin-containing regimen or without a parenteral amikacin or streptomycin-containing regimen?

Background: MAC isolates are usually susceptible in vitro to amikacin. Streptomycin was used in early noncomparative treatment trials during the initial months of treatment for both cavitary and nodular/ bronchiectatic MAC pulmonary disease [83, 138]. Parenteral aminoglycoside therapy was recommended in some previous NTM guidelines during the initial months of MAC therapy [152]. In the 2007 guideline [4], parenteral aminoglycosides were recommended for initial therapy of fibrocavitary MAC pulmonary disease and severe or previously treated MAC pulmonary disease [4]. Amikacin or streptomycin administration have been viewed as an intensification of oral therapy although that assumption has not been rigorously tested.

\section{Recommendation}

1) For patients with cavitary or advanced/severe bronchiectatic or macrolide-resistant MAC pulmonary disease, we suggest that parenteral amikacin or streptomycin be included in the initial treatment regimen (conditional recommendation, moderate certainty in estimates of effect).

Summary of the evidence: One randomized controlled trial was performed evaluating the impact of streptomycin addition to macrolide-based oral therapy for the initial three months of therapy [121]. One hundred forty-six patients with MAC pulmonary disease (both nodular/bronchiectatic and cavitary disease) were randomized to receive clarithromycin, ethambutol, and a rifamycin daily with (73) or without (73) streptomycin ( $15 \mathrm{mg} \cdot \mathrm{kg}^{-1} 3$ times per week during the initial 3 months of therapy). The sputum culture conversion rate was significantly higher for patients who received streptomycin than for those who received oral therapy only ( $71.2 \%$ versus $50.7 \%)$. There were, however, no significant differences in microbiologic recurrence rates or clinical improvement (which included both clinical symptoms and radiological findings). There were also no significant differences in adverse reactions and abnormal laboratory findings between the 2 groups. Two additional retrospective studies have suggested that the inclusion of a parenteral aminoglycoside administered for $\geqslant 6$ months in addition to adjunctive surgery improves outcome for patients with macrolide-resistant MAC pulmonary disease [16, 18]. There are no published data examining the relative efficacy of streptomycin versus amikacin for treating MAC pulmonary disease; streptomycin is no longer available in several countries. The relative and absolute effect estimates and 95\% CIs for each outcome (table E3.5) and discussion of value preferences, feasibility, cost, acceptability, and health inequality (table E4.5) can be found in the supplement.

Justification and implementation considerations: In the absence of comparably effective oral medications there are few options other than parenteral aminoglycosides for "intensifying" standard oral MAC therapy. Although the evidence is limited, it appears that there is some improvement in microbiologic response with the addition of three months of streptomycin to macrolide-based oral MAC therapy [121] and when administered for a longer duration in the setting of macrolide resistant MAC pulmonary disease [16, 18]. Amikacin must be paired with adequate companion medications, such as a macrolide, ethambutol and possibly rifampicin and clofazimine, to prevent the emergence of acquired mutational resistance and predictable treatment failure [153]. Based on the results of one randomized trial [121] and the experiences 
of the panel members, the benefits were felt to outweigh risks in those patients with cavitary or advanced/ severe bronchiectatic disease or those with macrolide-resistant MAC pulmonary disease. Administration of at least 2-3 months of an aminoglycoside was considered the best balance between risks and benefits.

Question VI. In patients with macrolide-susceptible MAC pulmonary disease, should a regimen with inhaled amikacin or a regimen without inhaled amikacin be used for treatment?

Background: Amikacin is active against MAC and has been recommended for intravenous treatment of cavitary or severe bronchiectatic MAC pulmonary disease [4]. However, systemic use of parenteral amikacin has been associated with a high frequency of renal, auditory, and vestibular toxicity [154]. Delivery of amikacin by hand-held nebulization may be a potential way to improve efficacy and decrease drug-related toxicity.

\section{Recommendations}

1) In patients with newly diagnosed MAC pulmonary disease, we suggest neither inhaled amikacin (parenteral formulation) nor ALIS be used as part of the initial treatment regimen (conditional recommendation, very low certainty in estimates of effect).

2) In patients with MAC pulmonary disease who have failed therapy after at least 6 months of guideline-based therapy, we recommend addition of ALIS to the treatment regimen instead of a standard oral regimen, only (strong recommendation, moderate certainty in estimates of effect).

Summary of the evidence: Reports evaluating the use of inhaled amikacin as part of a multidrug regimen for NTM pulmonary disease, including patients with MAC pulmonary disease, have primarily targeted patients with treatment refractory disease. Five retrospective case series ( $\mathrm{N}=138$ patients, 55 with MAC) with no comparator arm most commonly used inhaled doses of commercially available amikacin (parenteral formulation) ranging from 250 to $500 \mathrm{mg}$ once daily up to $15 \mathrm{mg} \cdot \mathrm{kg}^{-1}$ once daily added to their oral antibiotic regimen [155-159]. Clinical responses were reported in $20-100 \%$ and sputum conversion was reported in $18-67 \%$ of treatment refractory MAC pulmonary disease. Reported side effects in these series ranged from 8 to $38 \%$ and included hoarseness, throat irritation, bitter taste, and thrush. Ototoxicity occurred in 0 to $19 \%$ of patients with nephrotoxicity reported in only 1 patient and vertigo in 2 patients [155-159]. The relative and absolute effect estimates and 95\% CIs for each outcome (table E3.6) and discussion of value preferences, feasibility, cost, acceptability, and health inequality (table E4.6) can be found in the supplement.

A Phase II controlled trial randomized treatment refractory patients (e.g. with culture positivity after at least 6 months of guideline-based treatment that included a macrolide) with predominantly MAC $(n=57)$ or M. abscessus $(\mathrm{n}=32)$ pulmonary disease to investigational ALIS $(\mathrm{n}=44)$ versus placebo (empty liposomes, $n=45$ ) [19]. Although the primary endpoint of reduction in semiquantitative mycobacterial culture growth from baseline was not achieved, significantly more patients who received ALIS achieved culture conversion by day 84 and had greater improvement in distance achieved on 6-minute walk test. Adverse events were common ( $90 \%)$ in both groups, but patients receiving ALIS had more dysphonia and oropharyngeal discomfort, cough, wheezing, chest discomfort, acute exacerbations of bronchiectasis, and fatigue [19].

A randomized controlled phase III trial recently reported that ALIS, when added to guideline-based regimen for treatment refractory MAC pulmonary disease, was associated with a higher proportion of patients with negative cultures at 6 months compared to those who continued to take the standard regimen only [20]: Culture conversion was achieved by 65 of 224 patients (29.0\%) with ALIS + guideline-based therapy (GBT) compared with 10 of 112 (8.9\%) with GBT alone (odds ratio, 4.22; 95\% CI 2.08, 8.57; $\mathrm{p}<0.001)$. Adverse reactions were very common in both treatment arms: treatment-emergent adverse events (TEAE) were reported in $98.2 \%$ and $91.1 \%$ of patients in the ALIS+GBT and GBT-alone arms, respectively. The most common TEAEs overall were respiratory events reported by $87.4 \%$ and $50.0 \%$ of patients in the ALIS+GBT and GBT alone arms, respectively. TEAEs reported in $\geqslant 10 \%$ of patients in the ALIS+GBT arm included dysphonia, cough, hemoptysis, dyspnea, fatigue, diarrhea, nausea, and oropharyngeal pain. These events infrequently led to early discontinuation of ALIS (dyspnea, 3.1\%; dysphonia, 2.2\%; all others $<1 \%$ ) or withdrawal from the study. Audiological TEAEs were generally similar in both arms although tinnitus was reported in 17 patients $(7.6 \% ; 20$ events) in the ALIS+GBT arm compared with one event $(0.9 \%)$ in those receiving GBT alone. Vestibular TEAEs (dizziness, balance disorder, vertigo), although infrequent, were also more common in the ALIS+GBT arm than in the GBT alone arm. Serious TEAEs were reported in 45 patients (20.2\%) and 20 patients (17.9\%) in the ALIS+GBT and GBT-alone arms, respectively. During the study, more patients in the ALIS+GBT arm had MAC isolates with post-baseline amikacin MIC $>64 \mu \mathrm{g} \cdot \mathrm{mL}^{-1}$ than those receiving GBT alone $(10.3 \%$ versus $2.7 \%$ ). Of these $26.9 \%$ subsequently had MAC isolates with an MIC less than $64 \mathrm{mg} \cdot \mathrm{mL}^{-1}$. Based on the 
phase II and III trial results, ALIS was approved by the US Food and Drug Administration for treatment of MAC pulmonary disease in patients who have failed therapy after at least 6 months of GBT.

Justification and implementation considerations: There are insufficient data to support the use of inhaled antibiotics as an initial treatment option. There may be a risk of developing acquired mutational amikacin resistance with either inadequate companion medications or poor and irregular antibiotic deposition in the lung with areas of low amikacin concentration. In patients who fail treatment with an initial MAC regimen, inhaled therapy should be used as part of a salvage regimen to aggressively treat MAC pulmonary disease in those whose isolates retain in vitro susceptibility to amikacin. The results of phase II and phase III randomized trials $[19,20]$ of ALIS show that addition of ALIS to patients with MAC pulmonary disease that failed to convert sputum cultures after 6 months of GBT leads to culture conversion in $29 \%$ of patients in comparison to $9 \%$ in patients who continue GBT only. Because $10 \%$ of patients in the ALIS-arm developed amikacin resistance, the addition of another companion drug to prevent resistance development needs to be considered in these patients, although the preventive effect of an additional medication has not been determined in this situation. Where ALIS is not yet available, addition of inhaled parenteral amikacin is a reasonable alternative.

Question VII. In patients with macrolide-susceptible MAC pulmonary disease, should a 3-drug or a 2-drug macrolide-containing regimen be used for treatment?

Background: The poor response to treatment in AIDS patients with disseminated MAC in the premacrolide era and the rapid development of resistance with clarithromycin monotherapy reinforced the need for multiple drugs for treatment success. In contrast to the need for multidrug therapy, there is an opposing pressure to reduce the number of agents in MAC regimens to minimize drug-related adverse effects, the cost of the drug regimen, and the pill burden seen with 12-18 months of therapy.

\section{Recommendation}

1) In patients with macrolide-susceptible MAC pulmonary disease, we suggest a treatment regimen with at least 3 drugs (including a macrolide and ethambutol) over a regimen with 2 drugs (a macrolide and ethambutol alone) (conditional recommendation, very low certainty in estimates of effect).

Summary of the evidence: There are 2 randomized studies that compared a 2-drug regimen with a 3-drug regimen [21, 119], but only 1 of these studies included a macrolide-containing regimen [21]. In this single centre open label study from Japan, patients with previously untreated nodular/bronchiectatic or fibrocavitary MAC pulmonary disease were randomly assigned to either a daily 3-drug (clarithromycin/ ethambutol/rifampicin) or a daily 2-drug (clarithromycin/ethambutol) regimen for 12 months [21]. The drug doses (especially clarithromycin at $200 \mathrm{mg} 3$ times daily or twice daily based on body weight) were all lower than ATS/IDSA recommended dosing. The primary endpoint was sputum conversion (i.e. 3 consecutive negative cultures). Fifty-nine patients were assigned to a 3-drug regimen and 60 to a 2 -drug regimen with lung cavitation present in approximately $50 \%$ of patients in both arms. In the intent to treat analysis, the sputum culture conversion rate was $40.6 \%$ with the 3 -drug regimen and $55.0 \%$ with the 2 -drug regimen. The incidence of adverse events leading to the discontinuation of treatment was $37.2 \%$ and $26.6 \%$ for the 3-drug and the 2-drug regimens, respectively. In the per protocol analysis (those who completed therapy) 24/32 (75\%) converted on 3 drugs, and 33/40 (82.5\%) converted on 2 drugs. No isolates in either group developed macrolide resistance, although the study was underpowered to detect a difference. This study has significant limitations making interpretation difficult. The study was unblinded with a small sample size, had significant drop out during the course of the study, and used low doses of clarithromycin administered in a nonstandard frequency of dosing [160]. When combined with rifampicin in the 3-drug regimen, this would have led to low and potentially ineffective clarithromycin levels. The relative and absolute effect estimates and 95\% CIs for each outcome (table E3.7) and discussion of value preferences, feasibility, cost, acceptability, and health inequality (table E4.7) can be found in the supplement.

Justification and implementation considerations: A priority in MAC pulmonary disease therapy is preventing the development of macrolide resistance. Ethambutol is the best companion drug for preventing the emergence of macrolide resistance $[16,18,161]$. A 2-drug regimen including a macrolide and ethambutol is the regimen with the fewest possible drugs for treating MAC. The role of a rifamycin, or another third drug, is unclear. One possibility is that a third drug provides additional protection to that provided by ethambutol for preventing the emergence of macrolide resistance. In a randomized controlled trial of rifabutin added to clarithromycin and ethambutol for treatment of disseminated MAC infection, response rates, with or without rifabutin, were equivalent but development of macrolide resistance was lower $(\mathrm{p}=0.055)$ in patients on the 3-drug regimen [161]. Until additional evidence is provided showing that acquired macrolide resistance is equally common among macrolide containing 3-drug and 2 drug 
regimens, the panel prefers a 3-drug regimen. A PCORI-funded randomized controlled trial to evaluate the safety and efficacy of a 2 versus 3 drug regimen is currently underway (https://www.pcori.org).

Question VIII. In patients with macrolide-susceptible MAC pulmonary disease, should a daily or 3-times weekly regimen be used for treatment?

Background: The intermittent administration of antimycobacterial drugs has been a standard approach to drug susceptible tuberculosis therapy in North America for more than 2 decades [162]; therefore, it seems reasonable that macrolide susceptible MAC pulmonary disease might also be effectively treated with intermittent antibiotic administration. In the prior guideline [4], 3 times weekly therapy was recommended for patients with nodular/bronchiectatic MAC pulmonary disease but was not recommended for patients with cavitary disease, patients previously treated, or patients with moderate or severe disease $[4,163]$.

\section{Recommendations}

1) In patients with noncavitary nodular/bronchiectatic macrolide-susceptible MAC pulmonary disease, we suggest a 3 times per week macrolide-based regimen rather than a daily macrolide-based regimen (conditional recommendation, very low certainty in estimates of effect).

2) In patients with cavitary macrolide-susceptible MAC pulmonary disease we suggest a daily macrolide-based regimen rather than 3 times per week macrolide-based regimen (conditional recommendation, very low certainty in estimates of effect)

Summary of the evidence: No randomized trials have been performed that address this question; however, there are several cohort studies that have reported treatment outcomes with intermittent therapy. The first prospective noncomparative case series of patients receiving intermittent azithromycin-containing therapy for MAC pulmonary disease was reported in 1998 [164]. These preliminary results were followed by the results of 3 prospective noncomparative studies of azithromycin-containing regimens (including rifabutin or rifampicin, and ethambutol) for MAC pulmonary disease [140]. Patients received either intermittent azithromycin with daily companion medications, intermittent azithromycin with intermittent companion medications, or daily azithromycin with daily companion medicines. Conversion of sputum cultures to negative was observed in $17 / 29$ (59\%), 11/20 (55\%), and 28/43 (65\%) of patients, respectively. The microbiologic outcomes for the 3 regimens were not significantly different. In a subsequent study, 41 patients completed 6 months of therapy with clarithromycin $1000 \mathrm{mg}$, rifabutin 300-600 mg, and ethambutol $25 \mathrm{mg} \cdot \mathrm{kg}^{-1}$ administered 3 times per week [139]. Thirty-two (78\%) of these patients converted sputum cultures to negative. Adverse events associated with this regimen were primarily due to rifabutin, and in $41 \%$ of patients the dosage was decreased or the drug discontinued. These initial 3 studies included both cavitary and nodular bronchiectatic MAC pulmonary disease patients [139, 140, 164].

A large retrospective case series that included 180 patients with nodular/bronchiectatic MAC pulmonary disease reported outcomes with either daily or intermittent macrolide-containing (either azithromycin or clarithromycin) regimens (with rifampicin and ethambutol) for a minimum of 12 months [22]. Conversion of sputum cultures to negative occurred in $147 / 172$ (85\%) of patients treated with the intermittent regimen compared to 7 of 8 (88\%) patients who completed therapy with daily medication. A significantly greater number of patients treated with daily medications experienced medication intolerance and required a switch in regimen to intermittent therapy. None of the NTM strains from patients in the study developed macrolide resistance. Another retrospective study compared daily (earlier temporal period, 99 patients) with intermittent (later temporal period, 118 patients) administration of clarithromycin, rifampicin, and ethambutol for nodular/bronchiectatic MAC pulmonary disease [23]. Significantly more patients on daily therapy required regimen modification because of medication intolerance than patients on intermittent therapy (46\% versus 21\%). Seventy-six percent of patients receiving daily therapy, and $67 \%$ of patients receiving intermittent therapy converted cultures to negative. Acquired macrolide resistance was not reported in the study.

In addition to the 2 recent studies showing that intermittent macrolide-containing regimens are better tolerated than daily regimens, there may be other benefits to intermittent regimens. A case series suggested that intermittent ethambutol administration was less often associated with ethambutol-related ocular toxicity than daily ethambutol administration [165]. A recent systematic review reported that the default rate was $12.0 \%$ (95\% CI $8.9 \%-15.0 \%)$ in patients receiving 3 times weekly therapy compared to $16.0 \%$ (95\% CI 12.3-19.7\%) with daily administration [166]. A small study from South Korea on patients who were failing an intermittent regimen after 12 months of treatment reported that sputum culture conversion to negative was observed in approximately $30 \%$ of patients after switching to daily therapy [167].

Treatment outcomes with intermittent therapy are not as favourable in patients with cavitary pulmonary disease. A prospective open label multicentre trial reported a low culture conversion rate in patients with 
MAC pulmonary disease treated with 3 times weekly therapy [163]. Sputum culture conversion occurred in only $4 \%$ of patients with cavitary disease. Patients with noncavitary disease were approximately 4 times more likely than patients with cavitary disease to demonstrate sputum culture conversion and high-resolution computed tomography (CT), or symptom improvement. A recent case series from South Korea reported a high sputum culture conversion rate in patients with recurrent nodular/bronchiectatic disease who received an intermittent macrolide-based regimen [168]. In this case series, $86 \%$ of the recurrences were likely due to reinfection which would possibly explain the good outcomes. The relative and absolute effect estimates and 95\% CIs for each outcome (table E3.8) and discussion of value preferences, feasibility, cost, acceptability, and health inequality (table E4.8) can be found in the supplement.

Justification and implementation considerations: These recommendations are based on several noncomparative case series with consistent microbiologic results showing that intermittent therapy is similar to daily therapy for nodular/bronchiectatic MAC pulmonary disease and also better tolerated than daily therapy. A critically important finding from the available studies is the lack of development of macrolide resistance with intermittent therapy $[22,23]$. There is not similar evidence to justify or support intermittent therapy for cavitary MAC pulmonary disease and it is not recommended.

Question IX. In patients with macrolide-susceptible MAC pulmonary disease, should patients be treated with $<12$ months of treatment after culture negativity or $\geqslant 12$ months of treatment after culture negativity?

Background: Although MAC species are the most common organisms causing NTM pulmonary disease, the optimal treatment duration for MAC pulmonary disease has not been evaluated in a prospective randomized clinical trial. Although the duration of treatment of MAC pulmonary disease that is needed to achieve relapse-free cure is likely highly variable among individual patients, clinical guidance is needed for the recommendation of a general treatment duration.

\section{Recommendation}

1) We suggest that patients with macrolide-susceptible MAC pulmonary disease should receive treatment for at least 12 months after culture conversion (conditional recommendation, very low certainty in estimates of effect).

Summary of the evidence: There are no randomized studies or case series that address this question although there is one study that reported outcomes based on whether the patient received $<12$ months of treatment [22]. In a single centre retrospective observational cohort study that evaluated and reported treatment outcomes of patients with nodular/bronchiectatic MAC pulmonary disease, 27 patients received treatment for $<12$ months and 180 patients for $\geqslant 12$ months of a clarithromycin or azithromycin-based combination therapy, either daily or 3 times a week. Sputum culture conversion to negative was observed in 6 of the 27 patients (22\%) who received treatment for $<12$ months, compared with 154 of $180(86 \%)$ of patients who completed at least 12 months of therapy $(\mathrm{p}<0.001)$. The relative and absolute effect estimates and 95\% CIs for each outcome (table E3.9) and discussion of value preferences, feasibility, cost, acceptability, and health inequality (table E4.9) can be found in the supplement.

A recent systematic review reported that treatment success was higher in persons who received at least 12 months of macrolide-based therapy compared with $<12$ months [134]. Neither the aforementioned study nor the systematic review evaluated treatment outcomes by duration of treatment after culture conversion [134]. In a postmarketing study from Japan, bacteriologic relapse was noted in $5 \%$ of patients when treatment was continued for $<15$ months after sputum culture conversion and in zero patients who continued treatment for $>15$ months [136]. Given the lack of data on the optimal duration of therapy, the panel voted unanimously to continue to follow the recommendations from the 2007 guideline.

Justification and implementation considerations: The optimal duration of therapy for MAC pulmonary disease is currently not known. Semiquantitative sputum culture scores from the third month of treatment onwards are predictive of sustained sputum conversion at 12 months of treatment, so regular (e.g. monthly) sputum cultures are recommended during the treatment of MAC pulmonary disease [169]. There is currently not sufficient evidence to support bronchoscopy to obtain specimens for mycobacterial culture to determine the duration of therapy. Treatment outcome definitions have now been published to promote uniform outcome reporting in studies and gather more reliable data on optimal duration of therapy in MAC pulmonary disease [170]. In patients who fail to convert sputum cultures to negative after 6 months of treatment or who have extensive disease, expert consultation should be obtained. 
Treatment of MAC pulmonary disease: summary

We recommend a 3-drug, macrolide-based regimen for patients with macrolide-susceptible MAC pulmonary disease (tables 3 and 4). For patients with cavitary or advanced/severe bronchiectatic or macrolide-resistant MAC pulmonary disease, we suggest that parenteral amikacin or streptomycin be included in the initial treatment regimen. The parenteral agent is typically administered for at least 23 months. We suggest a 3 times per week regimen in patients with nodular/bronchiectatic disease but a daily macrolide-based regimen in those with cavitary disease. We suggest that treatment be administered for at least 12 months after culture conversion. If sputum cultures have not converted to negative after 6 months of guideline-based treatment, we recommend the use of ALIS as part of the continuation treatment regimen. In the setting of disease caused by macrolide-resistant MAC, the expert panel suggests seeking expert consultation.

\section{Treatment of M. kansasii pulmonary disease (Questions X-XIV) \\ Question X. In patients with rifampicin-susceptible M. kansasii pulmonary disease, should an isoniazid-containing regimen or a macrolide-containing regimen be used for treatment?}

Background: M. kansasii was one of the first NTM to be recognized to cause pulmonary disease [171]. Initially, a M. tuberculosis-like regimen including isoniazid was used, but treatment success was unsatisfactory $[30,172]$ until the introduction of rifampicin $[29,31]$. Once rifampicin was included in the regimen, treatment outcomes improved dramatically, and thus a rifampicin-based regimen is recommended [4]. Because of the uncertain value of isoniazid [173] and excellent in vitro activity of the macrolides [174-177], some clinicians have begun to substitute a macrolide for isoniazid in rifampicin-containing regimens [178].

\section{Recommendation}

1) In patients with rifampicin-susceptible $M$. kansasii pulmonary disease, we suggest a regimen of rifampicin, ethambutol, and either isoniazid or macrolide (conditional recommendation, very low certainty in estimates of effect).

Summary of the evidence: No randomized clinical trials have directly compared an isoniazid-containing regimen with a macrolide-containing regimen, but there are case series that reported treatment outcomes of these regimens for treating M. kansasii pulmonary disease. A 3-drug regimen that includes isoniazid, rifampicin, and ethambutol was recommended in the 2007 guideline [4]. Treatment outcomes with the 3-drug regimen when administered for 9-18 months have been excellent with cure rates of $80-100 \%$ and low relapse rates of $2.5-6.6 \%$ when administered for at least 12 months [27-29].

Untreated strains of $M$. kansasii are susceptible to macrolides, as minimal inhibitory concentrations of clarithromycin for M. kansasii range from 0.125 to $0.25 \mu \mathrm{g} \cdot \mathrm{mL}^{-1}$ [176]. Two small retrospective cohort studies evaluated treatment outcomes of regimens that substituted clarithromycin for isoniazid and reported similar cure rates of $80-100 \%[25,26]$. Among subjects who completed the treatment regimen, cure was $100 \%$. Discussion of value preferences, feasibility, cost, acceptability, and health inequality (table E4.10) can be found in the supplement.

Justification and implementation considerations: Isoniazid is widely used at present for treatment of $M$. kansasii pulmonary disease, and in the experience of the expert panel, there have been good outcomes when using a regimen consisting of rifampicin, ethambutol, and isoniazid irrespective of the result of MICs for isoniazid and ethambutol [24]. Based on the in vitro activity of macrolides against M. kansasii, and 2 studies that demonstrated good treatment outcomes when clarithromycin was substituted for isoniazid $[25,26]$, the panel suggests that either isoniazid or a macrolide can be used in combination with rifampin and ethambutol.

Question XI: In patients with rifampicin-susceptible M. kansasii pulmonary disease, should parenteral amikacin or streptomycin be included in the treatment regimen?

Background: Amikacin or streptomycin is sometimes used for treating NTM pulmonary disease. Studies that included 2-3 months of streptomycin added to a multidrug oral regimen demonstrated high rates of culture conversion and cure in patients with $M$. kansasii pulmonary disease [28, 29, 179]. However, their use in $M$. kansasii disease has not been recommended since the introduction of highly effective rifampicin-based regimens [4, 152, 173].

\section{Recommendation}

1) We suggest that neither parenteral amikacin nor streptomycin be used routinely for treating patients with $M$. kansasii pulmonary disease (strong recommendation, very low certainty in estimates of effect). 
TABLE 3 Dosing guidelines for drugs used in the management of nontuberculous mycobacterial pulmonary disease

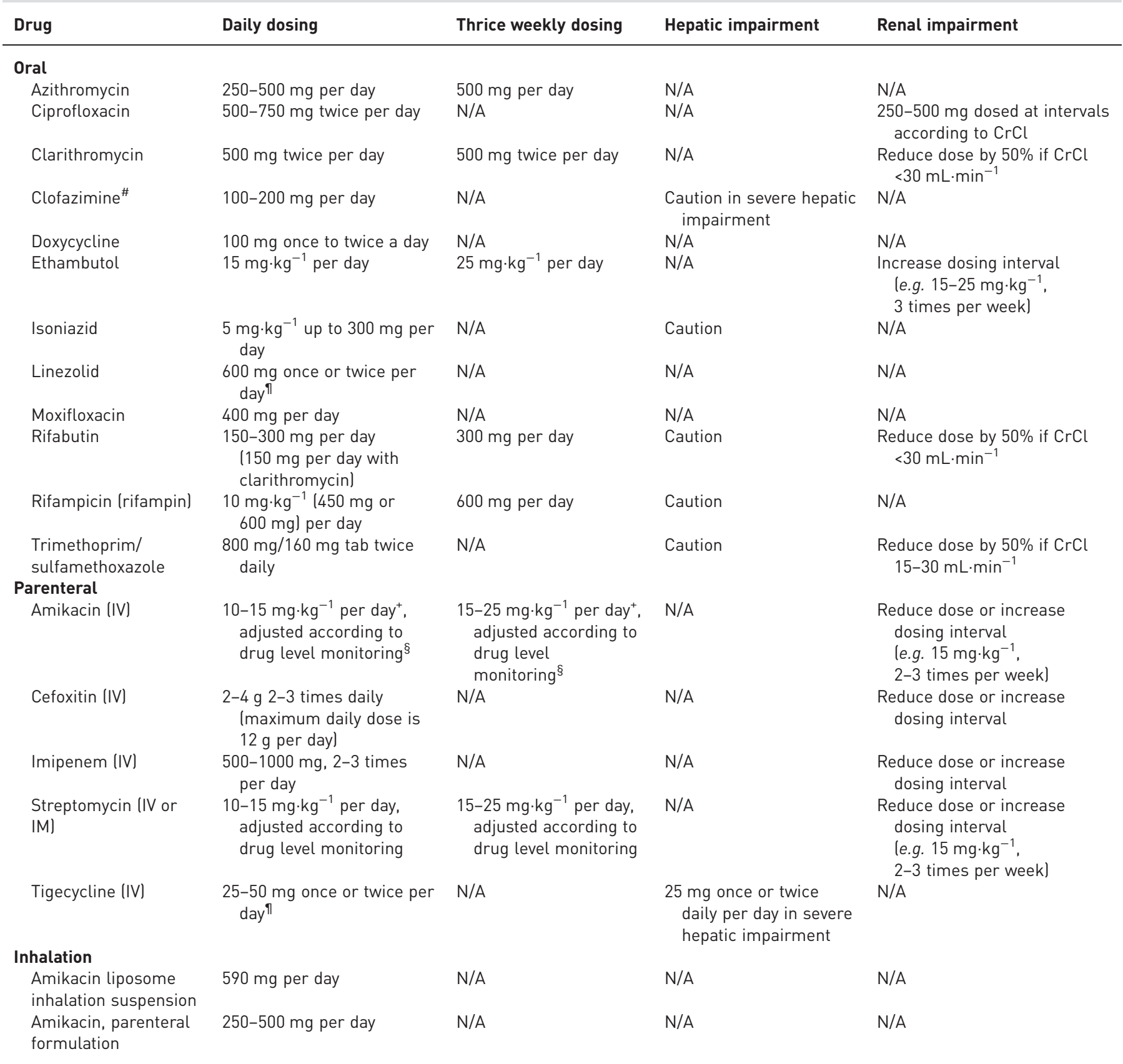

CrCL: creatinine clearance; IM: intramuscular; IV: intravenous; N/A: not applicable. ${ }^{\text {\# }}$ : clofazimine availability varies by country. In the United States, an investigational new drug application is required. ": most experts recommend once daily dosing of linezolid and tigecycline due to the high rate of drug-related adverse reactions associated with twice daily dosing. ${ }^{+}$: the use of the described regimens for 15 weeks was associated with permanent ototoxicity in approximately one third of patients, and the risk was associated with age and cumulative dose [154]. Given the high rates of ototoxicity, risks and benefits should be carefully considered in light of the goals of therapy. Clinicians should consider lower dose ranges and probably rely on intermittent dosing when more prolonged therapy is employed. \$: drug level monitoring: trough $<5 \mathrm{mg} \cdot \mathrm{L}^{-1}$; peak with daily dosing $35-45 \mu \mathrm{g} \cdot \mathrm{mL}^{-1}$; peak with intermittent dosing $65-80 \mu \mathrm{g} \cdot \mathrm{mL}^{-1}$ [154].

Summary of the evidence: There have been no randomized clinical trials addressing the use of amikacin or streptomycin for treating M. kansasii pulmonary disease, however three case series reported results with parenteral-containing regimens $[28,29,179]$. In one retrospective study including a mixture of NTM species, 16 patients with $M$. kansasii pulmonary disease were treated for 6 months to 2.5 years with regimens including streptomycin $(n=14)$ or capreomycin $(n=2)$ [179]. In the other 2 studies, 115 patients were treated with a rifampicin-based regimen that included isoniazid and ethambutol for 12 months, 
TABLE 4 Recommended treatment regimens for Mycobacterium avium complex, M. kansasii, and M. xenopi pulmonary disease

\begin{tabular}{|c|c|c|c|}
\hline Organism & Number of drugs & Preferred drug regimen" & Dosing frequency \\
\hline \multicolumn{4}{|l|}{ M. avium complex } \\
\hline Nodular-bronchiectatic & 3 & $\begin{array}{l}\text { Azithromycin (clarithromycin) } \\
\text { Rifampicin (rifabutin) } \\
\text { Ethambutol }\end{array}$ & 3 times weekly \\
\hline Cavitary & $\geqslant 3$ & $\begin{array}{l}\text { Azithromycin (clarithromycin) } \\
\text { Rifampicin (rifabutin) } \\
\text { Ethambutol } \\
\text { Amikacin IV (streptomycin) }{ }^{\pi}\end{array}$ & $\begin{array}{l}\text { Daily ( } 3 \text { times weekly may be used } \\
\text { with aminoglycosides) }\end{array}$ \\
\hline Refractory $^{+}$ & $\geqslant 4$ & $\begin{array}{l}\text { Azithromycin (clarithromycin) } \\
\text { Rifampicin (rifabutin) } \\
\text { Ethambutol } \\
\text { Amikacin liposome inhalation suspension } \\
\quad \text { or amikacin IV (streptomycin) }\end{array}$ & $\begin{array}{l}\text { Daily ( } 3 \text { times weekly may be used } \\
\text { with aminoglycosides) }\end{array}$ \\
\hline M. kansasii & 3 & $\begin{array}{l}\text { Azithromycin (clarithromycin) } \\
\text { Rifampicin (rifabutin) } \\
\text { Ethambutol }\end{array}$ & Daily \\
\hline & 3 & $\begin{array}{l}\text { Azithromycin (clarithromycin) } \\
\text { Rifampicin (rifabutin) } \\
\text { Ethambutol }\end{array}$ & 3 times weekly \\
\hline & 3 & $\begin{array}{l}\text { Isoniazid } \\
\text { Rifampicin (rifabutin) } \\
\text { Ethambutol }\end{array}$ & Daily \\
\hline
\end{tabular}

\section{M. xenopi}
Azithromycin (clarithromycin) and/or moxifloxacin Rifampicin (rifabutin)
Ethambutol
Amikacin"

Daily 13 times weekly may be used with aminoglycosides)

\footnotetext{
\#: see table 3 for recommended dosages. Alternative drugs for patients who are intolerant of or whose isolate is resistant to first-line drugs include clofazimine, moxifloxacin, and linezolid. Some experts would consider bedaquiline or tedizolid. ๆ: consider for cavitary, extensive nodular/ bronchiectatic disease or macrolide-resistant MAC. Amikacin or streptomycin may be given 3 times a week. ${ }^{+}$: refractory disease is defined as remaining sputum culture positive after 6 months of guideline-based therapy. Amikacin liposome inhalation suspension (ALIS) has been shown to improve culture conversion when added to guideline-based therapy in treatment refractory patients with MAC pulmonary disease.
}

supplemented with streptomycin 3 days a week for the first 2 months [29]. The pooled culture conversion rate was $95.5 \%$ (42 of 44 patients in 2 studies) [29, 179], and recurrences were observed in $4.7 \%$ (6 of 127 patients in 3 studies) $[28,29,179]$. Significant adverse events were reported in one study (14.7\%), leading to discontinuation of the parenteral agent in 9.5\% [28]. Studies that have used oral regimens without inclusion of aminoglycosides have also demonstrated high culture conversion rates and cure with low relapse rates [25-27]. The relative and absolute effect estimates and 95\% CIs for each outcome (table E3. 11) and discussion of value preferences, feasibility, cost, acceptability, and health inequality (table E4.11) can be found in the supplement.

Justification and implementation considerations: In general, regimens of 3 oral agents, rifampicin and ethambutol, and either isoniazid or a macrolide, achieve high rates of sustained culture conversion and treatment success in the treatment of M. kansasii pulmonary disease. Therefore, given the good outcomes observed with oral regimens, the lack of data supporting the benefit of amikacin or streptomycin, and the potential risk of adverse effects associated with amikacin or streptomycin, the panel members felt strongly that the use of these parenteral agents is not warranted, unless it is impossible to use a rifampicin-based regimen or severe disease is present.

Question XII. In patients with rifampicin-susceptible M. kansasii pulmonary disease, should a treatment regimen that includes a fluoroquinolone or a regimen without a fluoroquinolone be used?

Background: In vitro testing shows susceptibility of clinical $M$. kansasii isolates to fluoroquinolones [175, $177,180,181]$, and fluoroquinolones are currently recommended as part of a multidrug regimen to treat 
rifampicin-resistant M. kansasii pulmonary disease [4]. It is not known whether the in vitro activity translates into treatment success that would lead to a change in the current treatment recommendation.

\section{Recommendations}

1) In patients with rifampicin-susceptible M. kansasii pulmonary disease, we suggest using a regimen of rifampicin, ethambutol, and either isoniazid or macrolide instead of a fluoroquinolone (conditional recommendation, very low certainty in estimates of effect).

2) In patients with rifampicin-resistant M. kansasii or intolerance to 1 of the first-line antibiotics we suggest a fluoroquinolone (e.g. moxifloxacin) be used as part of a second-line regimen (conditional recommendation, very low certainty in estimates of effect).

Summary of evidence: Although there is good in vitro activity of the fluoroquinolones against M. kansasii, no randomized clinical trial or case series have been published in which a fluoroquinolone was used for the treatment of $M$. kansasii pulmonary disease. Discussion of value preferences, feasibility, cost, acceptability, and health inequality (table E4.12) can be found in the supplement.

Justification and implementation considerations: Treatment success of $M$. kansasii pulmonary disease with a rifamycin-based drug regimen is usually excellent but the optimal choice of companion drugs is not clear. Although ethambutol is usually the preferred companion drug, the choice of an additional companion drug may be isoniazid, a macrolide, or a fluoroquinolone. As there is more experience and better evidence for treatment regimens that include isoniazid or a macrolide as a companion drug, these drugs are preferred. For rifampicin-resistant disease, a regimen such as ethambutol, azithromycin, and a fluoroquinolone would be likely to lead to successful treatment.

Question XIII. In patients with rifampicin-susceptible M. kansasii pulmonary disease, should a 3 times per week or daily treatment regimen be used?

Background: A rifamycin-based multidrug regimen for treatment of $M$. kansasii pulmonary disease is associated with a high cure rate when administered daily for at least 12 months [25, 27, 182]. Three times weekly treatment has been used successfully in the treatment of noncavitary MAC pulmonary disease [22, 23] and may decrease side effects and increase tolerability without impacting treatment success in patients with M. kansasii pulmonary disease [26].

\section{Recommendations}

1) In patients with noncavitary nodular/bronchiectatic $M$. kansasii pulmonary disease treated with a rifampicin, ethambutol, and macrolide regimen, we suggest either daily or 3 times weekly treatment (conditional recommendation, very low certainty in estimates of effect).

2) In patients with cavitary M. kansasii pulmonary disease treated with a rifampicin, ethambutol, and macrolide-based regimen, we suggest daily treatment rather than 3 times weekly treatment (conditional recommendation, very low certainty in estimates of effect).

3) In all patients with $M$. kansasii pulmonary disease treated with an isoniazid, ethambutol, and rifampicin regimen, we suggest treatment be given daily rather than 3 times weekly (conditional recommendation, very low certainty in estimates of effect).

Summary of evidence: Treatment regimens using daily administration of rifampicin, isoniazid, and ethambutol are associated with high treatment success and low relapse rates [27-29]. There are no studies that have evaluated treatment outcomes of this regimen when given intermittently. In contrast, clarithromycin-based treatment regimens have been demonstrated to have similarly good success rates [25, 26], even when given 3 times per week (14/14 evaluable patients converted sputum cultures and remained relapse free after $46 \pm 8.0$ months); 9 of the 14 patients had cavitary disease [26]. The relative and absolute effect estimates and 95\% CIs for each outcome (table E3.13) and discussion of value preferences, feasibility, cost, acceptability, and health inequality (table E4.13) can be found in the supplement.

Justification and implementation considerations: Cavitary NTM pulmonary disease has higher morbidity and mortality and warrants a more aggressive treatment approach than noncavitary disease [163, 183]. It is unclear to what extent this principle applies to patients with M. kansasii pulmonary disease given that 3 times weekly treatment can be effective in patients with nodular/bronchiectatic or cavitary disease [26]. However, because there are no randomized trials available and the small size of the single study that evaluated 3 times weekly therapy, the panel did not feel that they could recommend intermittent therapy in the setting of cavitary disease until more evidence was available. Similarly, there are no data to support the use of isoniazid on a 3 times weekly basis in patients with M. kansasii pulmonary disease. 
Question XIV: In patients with rifampicin-susceptible M. kansasii pulmonary disease, should treatment be continued for $<12$ months or $\geqslant 12$ months?

Background: Treatment for M. kansasii pulmonary disease with a rifampicin-based regimen for at least 12 months after negative sputum cultures was recommended by the 2007 ATS treatment guideline [4]. However, data from several studies suggest that a 12-month fixed duration may be enough to cure most patients [27-29].

\section{Recommendation}

1) We suggest that patients with rifampicin-susceptible $M$. kansasii pulmonary disease be treated for at least 12 months (conditional recommendation, very low certainty in estimates of effect).

Summary of the evidence: There have been no randomized clinical trials comparing $<12$ months with $\geqslant 12$ months of treatment after culture conversion, but a 12-month fixed duration regimen was evaluated in 3 studies [27-29], and a 9-month regimen in one [173]. A clinical trial randomized 28 patients into 2 groups of 14: one group received rifampicin, isoniazid and ethambutol daily for 6 months, followed by rifampicin and isoniazid to complete 12 months (14 patients), and the other group completed 18 months (14 patients) [27]. After 12-30 months of follow-up, one patient in the 12-month arm (7\%) and none in the 18-month arm recurred after completing treatment. In a prospective study [29], 40 patients were treated with $1 \mathrm{~g}$ of streptomycin (twice weekly for the first 3 months) plus rifampicin, isoniazid, and ethambutol for 12 months. One patient (2.5\%) recurred 6 months after completing treatment. Using the same regimen in a series of 75 patients [28], 5 (6.6\%) recurred after a median follow-up of 41.5 months. The pooled recurrence rate from these 3 studies was $5.4 \%$ (7 of 129 patients) [27-29]. The British Thoracic Society evaluated a 9-month regimen with rifampicin and ethambutol in 115 patients in a prospective study [173]. Although conversion of sputum to negative was achieved in $99.4 \%$ of patients, $10 \%$ experienced disease recurrence. The relative and absolute effect estimates and 95\% CIs for each outcome (table E3.14) and discussion of value preferences, feasibility, cost, acceptability, and health inequality (table E4.14) can be found in the supplement.

Justification and implementation considerations: Current rifampicin-based treatment regimens are associated with a high rate of success if used for at least 12 months [27, 29]. Randomized controlled trials comparing shorter treatment regimens are currently lacking. Although some experts would favour 12 months of treatment after culture conversion, there is no evidence that relapses could be prevented with treatment courses longer than 12 months. Some of the reported relapses may actually be exogenous reinfections, as suggested by the long periods between treatment completion and recurrence [27, 173]. Therefore, the panel members felt that M. kansasii could be treated for a fixed duration of 12 months instead of 12 months beyond culture conversion. Because sputum conversion at 4 months of rifampicin-based regimens is usually observed [29-31], expert consultation should be obtained if cultures fail to convert to negative by that time.

\section{Treatment of M. kansasii pulmonary disease: summary}

We suggest a regimen of rifampicin, ethambutol, and either isoniazid or macrolide for patients with rifampicin-susceptible M. kansasii pulmonary disease (tables 3 and 4). Neither parenteral amikacin nor streptomycin are recommended for routine use in these patients. We suggest that patients with nodular/ bronchiectatic $M$. kansasii pulmonary disease receive either daily or 3 times weekly treatment when receiving a macrolide, rifampicin, and ethambutol. However, in patients with cavitary disease, the regimen should be administered daily. In addition, when patients are treated with a regimen that includes isoniazid, rifampicin, and ethambutol, we suggest treatment be given daily. In patients with rifampicin-resistant $M$. kansasii or intolerance to one of the first-line antibiotics we suggest a fluoroquinolone (e.g. moxifloxacin) be used as part of a second-line regimen. We suggest that all patients be treated for at least 12 months.

Treatment of M. xenopi pulmonary disease (Questions XV-XVIII)

Question XV. In patients with M. xenopi pulmonary disease, should a treatment regimen that includes a fluoroquinolone or a regimen without a fluoroquinolone be used?

Background: $M$. xenopi pulmonary disease is difficult to treat and associated with high all-cause mortality $[35,36,131,184,185]$ that is higher than other NTM species, with a 5 -year mortality of $51 \%$ and $43 \%$ in population-based studies from Denmark and Canada, respectively [34, 186]. The elevated mortality may be due to the underlying lung disease, frequent concomitant chronic pulmonary aspergillosis [187, 188], as well as frequent cavitation among patients with M. xenopi disease [189]. In vitro data suggest that MIC values of fluoroquinolones are low for M. xenopi: in vitro activity of moxifloxacin is equal to that of clarithromycin [190]. In murine models, adding either moxifloxacin or clarithromycin to a rifampicin-ethambutol combination leads to drug regimens of equal efficacy [191]. 


\section{Recommendation}

1) In patients with $M$. xenopi pulmonary disease, we suggest using a multidrug treatment regimen that includes moxifloxacin or a macrolide (conditional recommendation, low certainty in estimates of effect).

Summary of the evidence: There are 2 systematic reviews that have reported treatment outcomes of $M$. xenopi pulmonary disease, and both noted a wide range of drugs and regimens used [184, 185]. Only 1 randomized clinical trial has been published that compared ciprofloxacin with clarithromycin when added to rifampicin and ethambutol in patients with $M$. xenopi pulmonary disease [131]. In this study, 34 patients were treated with either ciprofloxacin $(n=17)$ or clarithromycin $(n=17)$ in addition to rifampicin and ethambutol. No significant differences were found between the 2 regimens in term of death, cure, recurrence or adverse effects. However, the power of the study was too low to conclude which regimen was best (only 34 patients and 2 events). Moreover, in this study that also included patients with M. avium or M. malmoense, adverse events were not reported separately for M. xenopi. Preliminary data from a study in France in which randomized patients received either moxifloxacin or clarithromycin plus ethambutol and rifampicin reported no difference in the treatment success between the study arms [33]. The relative and absolute effect estimates and 95\% CIs for each outcome (table E3.15) and discussion of value preferences, feasibility, cost, acceptability, and health inequality (table E4.15) can be found in the supplement.

Justification and implementation considerations: There is in vitro evidence that macrolides and fluoroquinolones are active against M. xenopi, whereas rifampicin and ethambutol are inactive in vitro alone and in combinations [32]. From this perspective, a multidrug regimen that utilizes a macrolide or fluoroquinolone would be likely more active.

Question XVI. In patients with M. xenopi pulmonary disease, should a 2-, 3-, or 4-drug regimen be used for treatment?

Background: Despite the poor prognosis of M. xenopi pulmonary disease, there are few studies available on optimal treatment [35]. Like in other NTM infections, a multidrug therapy is used to avoid selecting for drug resistance, but the optimal number and combination of drugs are not known.

\section{Recommendation}

1) In patients with $M$ xenopi pulmonary disease, we suggest a daily regimen that includes at least 3 drugs: rifampicin, ethambutol, and either a macrolide and/or a fluoroquinolone (e.g. moxifloxacin) (conditional recommendation, very low certainty in estimates of effect).

Summary of evidence: There are 2 systematic reviews that have reviewed treatment outcomes of M. xenopi pulmonary disease, and both noted a wide range of drugs and regimens used [184, 185]. The authors of these reviews were unable to recommend the optimal number of drugs to be used in the regimen, although in 1 review, fluoroquinolone-containing regimens were associated with a greater proportion of relapse-free success [185]. Two randomized controlled studies in patients with M. xenopi pulmonary disease were conducted by the British Thoracic Society $[36,119,131]$. The first study compared efficacy of a regimen containing rifampicin, ethambutol with or without isoniazid in 42 patients (20 versus 22) [36, 119]. No significant differences were found in terms of death, cure or recurrence between the 2 groups. Nevertheless, the power is probably insufficient, with few patients included and few events occurred. The main result of this study was the poor prognosis of these patients (5-year mortality of 57\% with M. xenopi versus $31 \%$ in MAC disease and $25 \%$ in $M$. malmoense disease). In the second study, 34 patients with $M$. xenopi pulmonary disease were randomized to receive rifampicin, ethambutol, and either ciprofloxacin or clarithromycin. Treatment failure/relapse occurred in $24 \%$ of the clarithromycin group versus $6 \%$ in the ciprofloxacin group [131]. In a murine model of $M$. xenopi infection, a 4-drug regimen (rifampicin, ethambutol, amikacin, and clarithromycin or moxifloxacin) demonstrated better efficacy than a 3-drug regimen (rifampicin, ethambutol, and moxifloxacin or clarithromycin) [191]. The relative and absolute effect estimates and 95\% CIs for each outcome (table E3.16) and discussion of value preferences, feasibility, cost, acceptability, and health inequality (table E4.16) can be found in the supplement.

Justification and implementation considerations: In animal and in vitro models, regimens of rifampicin, ethambutol, and either clarithromycin or moxifloxacin are efficacious and those that included amikacin (see Question 17) even more so. Given the very high mortality associated with M. xenopi, the committee felt the large risk of treatment failure with a 2-drug regimen warranted a strong recommendation for at least a 3-drug treatment regimen. However, the lack of confidence in the estimates of effect from the available studies tempered the recommendation. Additionally, the absence of universal access to 
moxifloxacin and the small amount of data for other fluoroquinolones has to be considered when choosing a regimen.

\section{Question XVII. In patients with M. xenopi pulmonary disease, should parenteral amikacin or} streptomycin be included in the treatment regimen?

Background: Patients with M. xenopi pulmonary disease frequently present with cavitary disease [189], often respond poorly to treatment $[35,36,184,185]$, and suffer a higher all-cause mortality than other NTM species [34, 186]. Based on expert opinion, the 2007 guideline suggested that adding streptomycin to a multidrug oral regimen is reasonable [4]. However, there is substantial uncertainty regarding best treatment regimens for M. xenopi.

\section{Recommendation}

1) In patients with cavitary or advanced/severe bronchiectatic $M$. xenopi pulmonary disease, we suggest adding parenteral amikacin to the treatment regimen and obtaining expert consultation (conditional recommendation, very low certainty in estimates of effect).

Summary of the evidence: For the current guideline, no high-quality studies addressing the question were identified. In a systematic review of $M$. xenopi pulmonary disease, data regarding parenteral therapy were found exclusively in retrospective series, and the data synthesis identified evidence against aminoglycosides [185]. Compared with patients who did not receive aminoglycosides, patients who received aminoglycosides had lower success rates both in the short term ( $56 \%$ versus $82 \%, \mathrm{p}=0.019)$ and long term ( $38 \%$ versus $68 \%, \mathrm{p}=0.029$ ). However, the comparison was undoubtedly biased strongly by disease severity. Two studies in mice infected with $M$. xenopi have shown reduced colony forming units among mice treated with amikacin in addition to comparator regimens [191, 192]. One study used intravenously infected mice treated with clarithromycin, ofloxacin plus/minus amikacin [192], and the other study used an inhalational infection model and treatment with either clarithromycin/ethambutol/rifampicin or moxifloxacin/ethambutol/rifampicin plus/minus amikacin [191], and both studies identified microbiologic benefit of the addition of amikacin. Discussion of value preferences, feasibility, cost, acceptability, and health inequality (table E4.17) can be found in the supplement.

Justification and implementation considerations: This recommendation is based on expert opinion and data from murine models of $M$. xenopi infection, wherein microbiologic benefit was observed in mice treated with amikacin [191, 192]. Barring compelling evidence to the contrary, M xenopi patients should be treated aggressively given the high mortality of the disease [34-36]. In addition to the high mortality, the panel considered the general acceptability and feasibility of parenteral therapy, and potential costs and toxicities, all based on clinical experience.

Question XVIII. In patients with M. xenopi pulmonary disease, should treatment be continued for $<12$ months or $\geqslant 12$ months after culture conversion?

Background: The optimal duration of treatment for M. xenopi pulmonary disease is not known, neither is the effect of treatment duration on the frequency of disease recurrence. The 2007 guideline suggested a treatment duration of 12 months beyond culture conversion, acknowledging that the optimal duration was unknown [4].

\section{Recommendation}

1) In patients with M. xenopi pulmonary disease, we suggest that treatment be continued for at least 12 months beyond culture conversion (conditional recommendation, very low certainty in estimates of effect).

Summary of the evidence: No studies have specifically addressed this question. Two studies in the 1980s found that treatment durations had an effect on outcomes (typically with isoniazid-rifampicin-ethambutol regimens). Treatment duration over 18 months lead to relapse-free cure in 8/11 patients [122]; treatment regimens over 9 months of duration cured more patients (11/23) than shorter regimens (1/11) [37]. A 2009 systematic review concluded that the data available at the time of the review did not permit comment on the impact of treatment duration on treatment outcomes [185]. Subsequent case series could not address the specific question but found that treatment duration of $<6$ months was associated with higher mortality and with recurrence [35]. One clinical trial has examined 24-month long regimens for M. xenopi pulmonary disease; 12 of 34 (35\%) patients treated showed a favourable response that could be sustained for 3 years after treatment; however, 18 patients (54\%) deviated from the treatment protocol, for which no further details are available [131]. Three retrospective case series have reported on outcomes and mean or median treatment duration, but regimens varied and none of these studies specifically correlated treatment duration with outcomes. A study in France recorded 27\% clinical and/ or microbiological conversion with 
a median duration of treatment of 5 months in 122 patients [35]. In Croatia, 6 months of first-line antituberculosis treatment led to favourable outcomes in 10 of 20 patients (50\%) [193]. In the Netherlands, 11 of 19 patients $(58 \%)$ treated for a mean of 9 months achieved culture conversion sustained until end of treatment [123]. Mortality rates varying from 21\% [123] to 41\% [131] and even 69\% [35] suggest that long-term treatment and follow-up are a significant challenge in this specific disease. The relative and absolute effect estimates and 95\% CIs for each outcome (table E3.18) and discussion of value preferences, feasibility, cost, acceptability, and health inequality (table E4.18) can be found in the supplement.

Justification and implementation considerations: The data reviewed above suggest that treatment outcomes improve if the duration of treatment increases. The panel members felt that this outweighs the risk of adverse events associated with longer treatment and agrees with previous recommendations [4].

\section{Treatment of M. xenopi pulmonary disease: summary}

In patients with $M$. xenopi pulmonary disease, we suggest a daily regimen that includes at least 3 drugs: rifampicin, ethambutol, and either a macrolide and/or a fluoroquinolone (e.g. moxifloxacin) (tables 3 and 4). In patients with severe $M$. xenopi pulmonary disease, we suggest adding parenteral amikacin to the treatment regimen and obtaining expert consultation given the poor treatment outcomes. We suggest treatment be continued for $\geqslant 12$ months after culture conversion.

\section{Treatment of M. abscessus pulmonary disease (Questions XIX-XXI)}

Question XIX. In patients with M. abscessus pulmonary disease, should a macrolide-based regimen or a regimen without a macrolide be used for treatment?

Background: Macrolides possess potent activity against M. abscessus as well as immunomodulatory effects. Macrolide resistance can develop through chromosomal mutations in the $23 \mathrm{~S} \mathrm{rDNA}(\mathrm{rrl})$ gene resulting in high level mutational resistance as well as through induction of the erm(41) gene that causes inducible resistance in the presence of a macrolide [125]. M. abscessus subsp. (abscessus, bolletii, and massiliense) are rapidly growing mycobacteria that differ in in vitro susceptibility to macrolides based on the functionality of the erm(41) gene [194]. The different mechanisms leading to macrolide resistance have made it difficult for clinicians to determine when to use a macrolide in the treatment of M. abscessus pulmonary disease.

\section{Recommendations}

1) In patients with M. abscessus pulmonary disease caused by strains without inducible or mutational resistance, we recommend a macrolide-containing multidrug treatment regimen (strong recommendation, very low certainty in estimates of effect).

2) In patients with $M$. abscessus pulmonary disease caused by strains with inducible or mutational macrolide resistance, we suggest a macrolide-containing regimen if the drug is being used for its immunomodulatory properties although the macrolide is not counted as an active drug in the multidrug regimen (conditional recommendation, very low certainty in estimates of effect).

Summary of evidence: There were no studies identified that compared macrolide-containing regimens with nonmacrolide-containing regimens. A recent systematic review [195] reported that a single study reported the use of macrolide-free regimens in 120 patients of whom $8 \%$ experienced culture conversion [196]. This review included an additional 13 studies that used macrolide-containing regimens of which 10 were retrospective [38, 39, 89, 197-203] and 3 prospective cohort designs [12, 108, 204]. A second systematic review [184] included 10 studies including 2 [90, 205] that were not assessed in the other systematic review. Evidence from these studies has demonstrated the importance of macrolide susceptibility and treatment outcomes. Compared with the macrolide-free regimen, the macrolide-containing regimens had a pooled sustained sputum culture conversion of $34 \%$ with $M$. abscessus subsp. abscessus and 54\% with subsp. massiliense [195]. Overall, good treatment outcomes were noted in $84 \%$ of those with M. abscessus subsp. massiliense compared with $23 \%$ with subsp. abscessus.

Four studies compared treatment outcomes in patients with infections due to M. abscessus subsp. abscessus or massiliense [38, 198, 199, 203, 206, 207]. Among the over 200 patients included in the studies, culture conversion ranged between $25-42 \%$ and $50-96 \%$ among those with subsp. abscessus and massiliense, respectively. The very large differences in culture conversion between the 2 subspecies were likely related to the nonfunctional erm(41) gene (no inducible resistance) in subsp. massiliense and a functional gene in most isolates of subsp. abscessus. This strongly suggests that macrolides provide a very large benefit in the treatment of macrolide-suspectible $M$. abscessus. Additional data demonstrating the importance of the macrolide in treatment is a study that reported that only 1 (7\%) patient with macrolide resistant $M$. abscessus subsp. massiliense had a favourable outcome with treatment [124]. The relative and absolute effect estimates and 95\% CIs for each outcome (table E3.19) and discussion of value preferences, feasibility, cost, acceptability, and health inequality (table E4.19) can be found in the supplement. 
Justification and implementation considerations: $M$. abscessus infections can be life-threatening, and the use of macrolides is potentially of great benefit. Macrolides are very active in vitro against M. abscessus strains without a functional erm(41) gene [208]. The far better treatment outcomes in studies of $M$. abscessus subsp. massiliense versus subsp. abscessus (inactive versus active erm(41) gene), where treatment differences appear to depend on the activity of the macrolide, strongly suggest a major benefit from this drug class [38, 39, 203, 206, 207]. Despite the very low certainty in the estimates of effect, the committee felt a strong recommendation was appropriate given the high morbidity and mortality of $M$. abscessus infections and significant potential clinical impact of macrolides given their in vitro activity.

It is important to consider identification of the M. abscessus subsp. in addition to in vitro macrolide susceptibility testing, because of the difference in response to macrolide therapy based on the presence of a functional or nonfunctional erm(41) gene. The acquisition of treatment associated mutational macrolide resistance in patients with $M$. abscessus, with or without inducible macrolide resistance, suggests that mutations in 23S rRNA are responsible for high level macrolide resistance [125]. In this setting, macrolides are unlikely to be contributing to the antimicrobial effect of the treatment regimen.

Macrolides have been demonstrated to prevent exacerbations of bronchiectasis in patients with chronic Pseudomonas infection, despite the lack of antimicrobial activity against Pseudomonas [209, 210], which is a common copathogen in patients with bronchiectasis [211]. However, the risk of acquiring resistance to other coinfecting pathogens must be considered when macrolides are used for immunomodulatory purposes in patients whose isolate has documented inducible or mutational macrolide resistance [209, 210]. As with all patients receiving treatment, frequent sputum cultures should be obtained during the course of therapy to monitor for treatment response and survey for the appearance of other organisms such as M. avium complex. In this setting, the treatment regimen should be adjusted to cover the new isolates in order to avoid development of macrolide resistance in the new NTM.

\section{Question XX. In patients with M. abscessus pulmonary disease, how many antibiotics should be included within multidrug regimens?}

Background: M. abscessus isolates display in vitro resistance to most oral antibiotics and are generally susceptible to a limited number of parenteral agents including tigecycline, imipenem, cefoxitin, and amikacin. Previous guidelines recommend using a multidrug regimen including $\geqslant 2$ of these antibiotics to which the organism is susceptible in vitro. Recent work suggests a lack of consensus among treating physicians, with a variety of regimens employed against this organism ranging from 2 to 5 drugs in the initial phases of therapy [212].

\section{Recommendation}

1) In patients with $M$. abscessus pulmonary disease, we suggest a multidrug regimen that includes at least three active drugs (guided by in vitro susceptibility) (conditional recommendation, very low certainty in estimates of effect).

Summary of the evidence: There are 2 systematic reviews [184, 195] that have reported treatment outcomes in patients with $M$. abscessus pulmonary disease, but there are no studies that have directly compared the efficacy or safety of different multidrug regimens. Based on the systematic reviews, the overall sputum culture conversion in patients with $M$. abscessus (not further subspeciated) treated with a multidrug, macrolide-containing regimen was 59\%: culture conversion occurred in $34-41 \%$ in those with M. abscessus subsp. abscessus and 54-69.8\% in those with M. abscessus subsp. massiliense [184, 195]. One observational retrospective study attempted to compare a macrolide plus amikacin regimen versus a 3-drug regimen consisting of a macrolide, amikacin, and either imipenem or cefoxitin [198]. However, they did not distinguish patients with M. abscessus isolates with and without functional erm genes. Accordingly, the interpretation of outcomes associated with these regimens was not possible. One additional observational retrospective study suggested that multidrug therapy is associated with improved quality of life in $M$. abscessus patients, but this study did not compare outcomes according to different drug regimens [108]. Importantly, the few cases series that have described treatment outcomes all used multidrug regimens with $\geqslant 3$ drugs $[184,195]$. The relative and absolute effect estimates and 95\% CIs for each outcome (table E3.20) and discussion of value preferences, feasibility, cost, acceptability, and health inequality (table E4.20) can be found in the supplement.

Justification and implementation considerations: Given the usual disease severity of $M$. abscessus pulmonary disease, the variable and limited in vitro drug susceptibility of these organisms, the potential for the emergence of drug resistance, and the potential for more rapid progression of $M$. abscessus pulmonary disease, the expert panel suggests using a regimen consisting of $\geqslant 3$ active drugs in macrolide susceptible disease and at least 4 drugs, when possible, in macrolide resistant disease. This is particularly true in the initial months of therapy when bacterial burdens are greater. Design of regimens beyond the 
initial intravenous phase is difficult given the lack of oral antimicrobials with activity against M. abscessus. Although macrolides might still be useful for immunomodulatory effects or antimicrobial effects against other coinfecting organisms, they are not counted as an active drug against $M$. abscessus when inducible or mutational resistance is noted. The committee members feel strongly that treatment regimens should be designed in collaboration with experts in the management of these complicated infections.

Question XXI. In patients with M. abscessus pulmonary disease, should shorter or longer duration therapy be used for treatment?

Background: The 2007 guideline noted that no medication strategy could reliably achieve the goal of 12 months of negative sputum cultures while on therapy [4]. It was therefore suggested that periodic treatment courses, or aggressive treatment regimens including multiple parenteral agents for a few months, could be effective strategies. However, the optimum treatment duration of pulmonary disease caused by $M$. abscessus complex is currently unknown.

\section{Recommendation}

1) In patients with M. abscessus pulmonary disease, we suggest that either a shorter or longer treatment regimen be used and expert consultation obtained (conditional recommendation for either the intervention or comparator, very low certainty in estimates of effect).

Summary of the evidence: Only 1 study addressing this specific question was identified by the systematic review [213]. This observational, retrospective study included 30 patients with M. abscessus pulmonary disease who met the diagnostic criteria defined in the 2007 guideline. Overall, 17 of the patients were treated for $>1$ month and had follow-up available for at least 1 year: 13 were treated for less than 12 months, and 4 were treated for $\geqslant 12$ months. No significant difference was found in the cure rate between the 2 groups. No additional information was available with regard to lung involvement, nor to the subsp. of $M$. abscessus. The study methodology, notably no control for confounding, indirect comparisons with different regimens of various duration, and a wide confidence interval, indicate high risk of bias. Two recent systematic reviews did not address the optimum duration of therapy but noted that most patients with $M$. abscessus were treated for over 12 months with multidrug regimens including a minimum of 4 weeks of $\geqslant 1$ parenteral antimicrobials $[184,195]$. The relative and absolute effect estimates and 95\% CIs for each outcome (table E3.21) and discussion of value preferences, feasibility, cost, acceptability, and health inequality (table E4.21) can be found in the supplement.

Given the better treatment outcomes with disease due to M. abscessus subsp. massiliense, a shorter or less intensive course of therapy may be possible. In a retrospective study of 128 patients with $M$. abscessus, patients with $M$. abscessus subsp. massiliense had better treatment outomes than patients with subsp. abscessus despite receiving shorter durations of parenteral and total treatment: patients with M. abscessus subsp. massiliense received a median of 4.7 months of parenteral therapy and 12.1 months of total treatment compared with 7.4 and 16.3 months in patients with $M$. abscessus subsp. abscessus, respectively [207]. In another study, 71 patients with M. abscessus subsp. massiliense were treated with either 2 or 4 weeks of intravenous amikacin and cefoxitin (or imipenem) along with an oral macrolide [204]. Those treated with a 2-week course of parenteral therapy followed by at least 12 months of an oral macrolide post conversion had a culture conversion rate of $91 \%$ compared with $100 \%$ in those who received a 4 -week course and oral macrolide for 24 months. Two patients who received the shorter course of therapy developed acquired macrolide resistance. Although the expert panel does not recommend macrolide monotherapy for treatment of NTM pulmonary disease, the study demonstrated that similar treatment outcomes could be obtained using shorter and less intensive treatment than used for M. abscessus subsp. abscessus.

Justification and implementation considerations: The 1 study identified had a very small sample size, only indirectly addressed this question, and was felt to be of too low quality to form the basis of a recommendation. The lack of studies evaluating treatment durations, the variation in drug and resource availability, as well as the diverse practice settings, made it difficult to come to a consensus on the optimum duration of therapy. In addition, the panel members felt that some subgroups of patients should be considered separately in determining the length of therapy such as: patients with nodular/bronchiectatic versus cavitary disease, patients affected by lung disease caused by different $M$. abscessus subspecies and, importantly, depending on susceptibility to macrolides and amikacin. Although the optimal duration of therapy is not known, most patients reported in the literature with $M$. abscessus were treated for $>12$ months, and the treatment was divided into an initial phase usually including parenteral drugs followed by a longer phase using oral and sometimes inhaled antibiotics [184, 195]. The panel members suggest that an expert in the management of patients with M. abscessus pulmonary disease be consulted prior to initiation of therapy in order to assist with determination of the duration of therapy. 
Treatment of M. abscessus pulmonary disease: summary

The optimal drugs, regimens, and duration of therapy are not known. Patients with M. abscessus pulmonary disease caused by strains without inducible (typically $M$. massiliense) or mutational macrolide resistance should be treated with a macrolide-containing multidrug regimen that includes at least 3 active drugs (guided by in vitro susceptibility) in the initial phase of treatment (the phase including intravenous agents) (tables 3 and 5). In patients with $M$. abscessus pulmonary disease caused by strains with inducible (typically $M$. abscessus or $M$. bolettii) or mutational macrolide resistance, we suggest a regimen that includes at least 4 active drugs, when possible. We suggest a macrolide-containing regimen if the drug is being used for its immunomodulatory properties although the macrolide is not counted as an active drug in the multidrug regimen. For the continuation phase of therapy (after the parenteral component), we suggest that at least 2-3 active drugs be given. Some experts would use intermittent courses of multidrug therapy instead of transitioning to a longer continuation phase, although almost all published studies treated patients for $>12$ months. In the absence of data to support a shorter or longer treatment course for M. abscessus pulmonary disease, the panel members suggest that expert consultation be obtained prior to initiation of therapy in order to assist with design of the regimen and determine whether a shorter or longer treatment regimen should be used.

\section{Surgical resection for treatment of NTM pulmonary disease (Question XXII) Question XXII. Should surgery plus medical therapy or medical therapy alone be used to treat NTM pulmonary disease?}

Background: NTM pulmonary disease is often difficult to cure with antimicrobial therapy alone. Selected patients with failure of medical management, cavitary disease, drug-resistant isolates, or complications such as hemoptysis or severe bronchiectasis may undergo surgical resection of the diseased lung. The decision to proceed with surgical resection must be weighed against the risks and benefits of surgery.

\section{Recommendation}

1) In selected patients with NTM pulmonary disease, we suggest surgical resection as an adjuvant to medical therapy after expert consultation (conditional recommendation, very low certainty in estimates of effect).

Summary of the evidence: We identified 15 observational studies [30, 39, 43, 89, 214-223] including approximately 700 patients who underwent various surgical resections including segmentectomies, lobectomies, and pneumonectomies. Most patients included in the studies had MAC pulmonary disease, with 1 study including only patients with $M$. xenopi pulmonary disease [221], 1 with $M$. kansasii only [30], and 2 including patients with $M$. abscessus pulmonary disease [39, 89]. Almost all of the patients who underwent surgery had received antimicrobial treatment before and after surgery. Three studies reported results for patients treated with combined antibiotic and surgical therapy, compared with antibiotic therapy alone [30, 39, 89].

Cure rate of the NTM disease, death, and recurrences were not significantly different between medical and surgical therapy in the 3 comparative studies that included a total of 296 patients with follow-up data (95 surgical plus medical and 201 medical only). Although there was more culture conversion observed in the patients who underwent surgery, the quality of evidence was very low, due to the small number of patients treated, inherent selection bias by treatment group, lack of adjustment for other clinical variables, and the fact that all patients were treated by medical therapy. The desirable anticipated effects were estimated to be moderate. Surgical complications (such as bronchopleural fistula, prolonged air leak, pneumonia) were observed in $7-35 \%$ of participants. There was no operative mortality and postoperative mortality was reported in $0-9 \%$ of patients. In 1 study that reported outcomes of patients who underwent video assisted thoracoscopic surgery (VATS), culture conversion occurred in $84 \%$ of the patients, postoperative complications occurred in $7 \%$ of patients, and there were no operative or postoperative deaths reported [216]. Undesirable effects were estimated as small, and the balance between desirable and undesirable probably favours the intervention. There was no evidence identified for costs, which were estimated as moderate with regard to the duration of the disease. Therefore, surgery was estimated as acceptable to key stakeholders and feasible.

Justification and implementation considerations: The studies differed by location, the age and gender of patients, and the mycobacterial species involved (M. avium [214, 218, 220, 222], M. kansasii [30], M. abscessus [39, 89], M. xenopi [221] or a mix of species [89, 215-217, 219, 220, 223]). Moreover, the studies suffer from multiple potential biases including different reasons for performing surgery, patient selection, and subjective assessment of postsurgical outcomes. Even so, surgical resection was associated with improved treatment outcomes and for most of the patients (85-100\%), conversion of sputum cultures to negative was observed after surgery. Therapy with antimicrobial agents continued during and after the 
TABLE 5 Treatment regimens for Mycobacterium abscessus by macrolide susceptibility (mutational and inducible resistance)

\begin{tabular}{|c|c|c|c|c|}
\hline \multicolumn{2}{|c|}{ Macrolide susceptibility pattern } & \multirow[t]{2}{*}{ Number of drugs ${ }^{+}$} & \multirow[t]{2}{*}{ Preferred drugs } & \multirow[t]{2}{*}{ Frequency of dosing } \\
\hline Mutational $^{\#}$ & Inducible & & & \\
\hline Susceptible & Susceptible & Continuation phase $\geqslant 2$ & $\begin{array}{l}\text { Parenteral (choose 1-2) } \\
\text { Amikacin } \\
\text { Imipenem (or Cefoxitin) } \\
\text { Tigecycline } \\
\text { Oral (choose 2) } \\
\text { Azithromycin (clarithromycin) })^{\S} \\
\text { Clofazimine } \\
\text { Linezolid } \\
\text { Oral/inhaled (choose 2-3) } \\
\text { Azithromycin (clarithromycin) })^{\S} \\
\text { Clofazimine } \\
\text { Linezolid } \\
\text { Inhaled amikacin }\end{array}$ & $\begin{array}{l}\text { Daily (3 times weekly } \\
\text { may be used for } \\
\text { aminoglycosides) }\end{array}$ \\
\hline Resistant & $\begin{array}{l}\text { Susceptible } \\
\text { or resistant }\end{array}$ & $\begin{array}{l}\text { Initial phase } \geqslant 4 \\
\text { Continuation phase } \geqslant 2\end{array}$ & $\begin{array}{l}\text { Parenteral (choose 2-3) } \\
\text { Amikacin } \\
\text { Imipenem (or Cefoxitin) } \\
\text { Tigecycline } \\
\text { Oral (choose 2-3) } \\
\text { Azithromycin (clarithromycin) } \\
\text { Clofazimine } \\
\text { Linezolid } \\
\text { Oral/inhaled (choose 2-3) } \\
\text { Azithromycin (clarithromycin) } \\
\text { Clofazimine } \\
\text { Linezolid } \\
\text { Inhaled amikacin }\end{array}$ & $\begin{array}{l}\text { Daily (3 times weekly } \\
\text { may be used for } \\
\text { aminoglycosides) }\end{array}$ \\
\hline
\end{tabular}

\footnotetext{
\#: mutational resistance: none present: isolate determined to be phenotypically susceptible at 3-5 days of incubation in culture. Present: isolate determined to be phenotypically resistant at 3-5 days of incubation or sequencing identifies $r r l$ mutation know to confer resistance. " : inducible resistance: functional erm(41) gene: isolate determined to be resistant after 14 days of incubation or sequencing identifies functional gene sequence. Nonfunctional erm(41) gene: isolate determined to be susceptible after 14 days of incubation or sequencing identifies truncated sequence or $\mathrm{C} 28$ mutation (in subspecies abscessus). ${ }^{+}$: initial phase refers to the time that the parenteral agents are being given. Continuation phase refers to the subsequent phase of therapy that typically includes oral antimicrobial agents sometimes paired with inhaled agents. $\S$ : azithromycin (clarithromycin) is active in this setting and should be used whenever possible. ${ }^{f}$ : azithromycin (clarithromycin) activity is unlikely but can be added for its immunomodulatory effects but should not be counted as active against M. abscessus with a functional erm(41) gene. In this setting, frequent sputum cultures should be obtained to detect potentially new organisms like M. avium complex.
}

surgery, and the activity of these agents varied with regard to the study and the species involved (e.g. clarithromycin was given in recent studies but not in the older ones). Many experts feel it is desirable to achieve at least smear conversion prior to surgical resection, and the panel suggests that surgery be performed by a surgeon experienced in performing surgery on patients with mycobacterial disease [43].

\section{Monitoring for response to therapy}

Clinical, radiographic, and microbiologic data should be collected in order to assess whether or not a patient is responding to therapy. Chest radiographs or chest CT imaging may be beneficial for defining a 
TABLE 6 Common adverse drug reactions and monitoring recommendations ${ }^{\#}$

\begin{tabular}{|c|c|c|}
\hline Drug & Adverse reactions & Monitoring \\
\hline Clarithromycin & $\begin{array}{l}\text { Gastrointestinal } \\
\text { Tinnitus/hearing loss } \\
\text { Hepatotoxicity } \\
\text { Prolonged QTC }\end{array}$ & $\begin{array}{l}\text { Clinical monitoring } \\
\text { Audiogram } \\
\text { Liver function tests } \\
\text { ECG (QTC) }\end{array}$ \\
\hline Clofazimine & $\begin{array}{l}\text { Tanning of skin and dry-ness } \\
\text { Hepatotoxicity } \\
\text { Prolonged QTC }\end{array}$ & $\begin{array}{l}\text { Clinical monitoring } \\
\text { Liver function tests } \\
\text { ECG (QTc) }\end{array}$ \\
\hline Doxycycline & $\begin{array}{l}\text { GI upset } \\
\text { Photosensitivity } \\
\text { Tinnitus/vertigo }\end{array}$ & $\begin{array}{l}\text { Clinical monitoring } \\
\text { Clinical monitoring } \\
\text { Clinical monitoring }\end{array}$ \\
\hline Isoniazid & $\begin{array}{l}\text { Hepatitis } \\
\text { Peripheral neuropathy }\end{array}$ & $\begin{array}{l}\text { Liver function tests } \\
\text { Clinical monitoring }\end{array}$ \\
\hline Linezolid & $\begin{array}{l}\text { Peripheral neuropathy } \\
\text { Optic neuritis } \\
\text { Cytopenias }\end{array}$ & $\begin{array}{l}\text { Clinical monitoring } \\
\text { Visual acuity and colour discrimination } \\
\text { Complete blood count }\end{array}$ \\
\hline Moxifloxacin & $\begin{array}{l}\text { Prolonged QTC } \\
\text { Hepatotoxicity } \\
\text { Tendinopathy }\end{array}$ & $\begin{array}{l}\text { ECG (QTC) } \\
\text { Liver function tests } \\
\text { Clinical monitoring }\end{array}$ \\
\hline Trimethoprim/sulfamethoxazole & $\begin{array}{l}\text { GI upset } \\
\text { Cytopenias } \\
\text { Hypersensitivity } \\
\text { Photosensitivity }\end{array}$ & $\begin{array}{l}\text { Clinical monitoring } \\
\text { Complete blood count } \\
\text { Clinical monitoring } \\
\text { Clinical monitoring }\end{array}$ \\
\hline Rifampicin (rifampin) & $\begin{array}{l}\text { Hepatotoxicity } \\
\text { Cytopenias } \\
\text { Hypersensitivity } \\
\text { Orange discolouration of secretions }\end{array}$ & $\begin{array}{l}\text { Liver function test } \\
\text { Complete blood count } \\
\text { Clinical monitoring }\end{array}$ \\
\hline Amikacin, streptomycin, tobramycin & $\begin{array}{l}\text { Vestibular toxicity } \\
\text { Ototoxicity } \\
\text { Nephrotoxicity } \\
\text { Electrolyte disturbances }\end{array}$ & $\begin{array}{l}\text { Clinical monitoring } \\
\text { Audiograms } \\
\text { BUN, creatinine } \\
\text { Calcium, magnesium, potassium }\end{array}$ \\
\hline Amikacin liposome inhalation suspension & $\begin{array}{l}\text { Dysphonia } \\
\text { Vestibular toxicity } \\
\text { Ototoxicity } \\
\text { Nephrotoxicity } \\
\text { Cough } \\
\text { Dyspnea }\end{array}$ & $\begin{array}{l}\text { Clinical monitoring } \\
\text { Clinical monitoring } \\
\text { Audiograms } \\
\text { BUN, creatinine } \\
\text { Clinical monitoring } \\
\text { Clinical monitoring }\end{array}$ \\
\hline Cefoxitin & $\begin{array}{l}\text { Cytopenias } \\
\text { Hypersensitivity }\end{array}$ & $\begin{array}{l}\text { Complete blood count } \\
\text { Clinical monitoring }\end{array}$ \\
\hline Imipenem & $\begin{array}{l}\text { Rashes } \\
\text { Cytopenias } \\
\text { Nephrotoxicity }\end{array}$ & $\begin{array}{l}\text { Clinical monitoring } \\
\text { Complete blood count } \\
\text { BUN/Creatinine }\end{array}$ \\
\hline
\end{tabular}


TABLE 6 Continued

\begin{tabular}{lll} 
Drug & Adverse reactions & Monitoring \\
\hline Tigecycline & Nausea/vomiting & Clinical monitoring \\
& Hepatitis/pancreatitis & Liver function tests, amylase/lipase
\end{tabular}

Monitoring frequency should be individualized based on treatment regimen, age, comorbidities, concurrent drugs, overlapping drug toxicities, and resources. BUN: blood, urea, nitrogen; ECG: electrocardiogram; GI: gastrointestinal; QTc: corrected QT. \#: the expert panel recommends that patients have a complete blood count, liver function tests, and metabolic panel every 1-3 months in patients on oral therapy and weekly when on intravenous therapy. radiographic response to therapy, although there can be wide variability in findings given the common occurrence of underlying lung disease. Because the duration of therapy is based on the time of culture conversion, frequent collection of sputum specimens is required in order to determine the recommended treatment duration. The expert panel would consider obtaining sputum specimens for culture every 12 months in order to document when sputum cultures become negative. Sputum should be induced with hypertonic saline if spontaneous sputum specimens cannot be collected. Bronchoscopy should only be considered in exceptional circumstances to determine whether culture conversion has occurred. In addition to microbiologic assessments, clinical and radiographic response to therapy should be used to determine if the patient is responding to therapy.

\section{Monitoring for adverse reactions}

The drugs used to treat NTM pulmonary disease are frequently associated with adverse reactions. A recent randomized clinical trial reported that $>90 \%$ of subjects in each arm reported a treatment emergent adverse reaction [20]. Therefore, educating patients regarding potential reactions and monitoring for them is an important component of management. Rapid identification and management of an adverse reaction is likely to decrease the risk of treatment for the patient and possibly improve the chances of treatment completion. Table 6 lists common adverse reactions associated with the drugs used to treat NTM pulmonary disease and an approach to monitoring. Unfortunately, there are no studies that have identified the optimum frequency or most cost-effective approach to monitoring for drug-related adverse reactions. Monitoring frequency should be individualized based on age, comorbidities, concurrent drugs, overlapping drug toxicities, and resources.

\section{Therapeutic drug monitoring}

Therapeutic drug monitoring (TDM) refers to the measurement of drug concentrations in serum specimens at some point after dosing to determine whether or not a specific target concentration has been obtained (table 3). There are no randomized trials that have determined the clinical utility of performing TDM. However, studies have documented significant reductions in serum drug concentrations of clarithromycin with concurrent use of rifampicin and to a lesser extent with rifabutin [145, 224, 225]. Two studies described the association of serum concentrations of macrolides and treatment outcomes. The first study reported no association between the serum concentration of clarithromycin and treatment outcomes [224], whereas the second study noted a correlation between the peak serum concentration (Cmax) of azithromycin and favourable treatment outcomes when administered daily $(250 \mathrm{mg})$ but not intermittently (500 mg) [226]. Experts would consider performing TDM in situations in which drug malabsorption, drug underdosing, or clinically important drug-drug interactions are suspected [227]. Examples of situations in which TDM may be useful include patients with delayed sputum culture conversion or treatment failure not explained by nonadherence or drug resistance, patients receiving amikacin or streptomycin therapy and thus at risk of ototoxicity and nephrotoxicity, and patients with medical conditions (e.g. reduced renal function) that are suspected of leading to subtherapeutic or toxic drug concentrations.

\section{Research priorities}

During the development of this guideline, research gaps were identified for each of the PICO questions. Not surprisingly, there were many gaps and needs identified related to the treatment of NTM pulmonary disease. Many of the research priorities relate to the need for new drugs, treatment regimens, shorter regimens, and better tolerated regimens. Evaluation of new drugs will require standardized case definitions, outcome measures, and comparator regimens, as well as the ability to conduct multicentre trials [228]. A recent publication produced consensus definitions of microbiologic and functional endpoints [170]. In addition, a recent report of patient research priorities highlighted the importance of including quality of 
life outcomes in addition to microbiologic assessments in clinical trials [229]. The interested reader is referred to a separate publication that will follow highlighting these research gaps and priorities.

Acknowledgements: The writing committee thanks Kevin Wilson and the staff from each society for their guidance during the development of the guideline, and the reviewers for their critical comments which improved the focus and clarity of the guideline. This guideline is dedicated to the memory of Won-Jung Koh, whose passion, leadership, and work led to evidence that helped to support recommendations in this guideline. His tireless effort to improve the diagnosis and treatment of NTM disease will never be forgotten.

Conflict of interest: C.L. Daley served on advisory committees for Cipla, Horizon, Insmed, Johnson \& Johnson, Matinas Biopharma, Otsuka America Pharmaceutical, Paratek, and Spero; received research support from Beyond Air, Insmed, and Spero; served as a consultant for Meiji. J.M. Iaccarino has nothing to disclose. C. Lange served as a speaker for Berlin Chemie, Chiesi, Gilead, Janssen, Lucane, and Novartis; served on an advisory committee for Oxford Immunotec. E. Cambau has nothing to disclose. R.J. Wallace Jr served as the director of a university clinical laboratory that does NTM identification, molecular strain comparison, and susceptibility testing; received research support from Insmed as mycobacterial reference laboratory for a trial of the inhaled liposomal amikacin. C. Andrejak received research support from Insmed. E.C. Böttger served as a consultant for AID Diagnostika, Becton Dickinson, and COPAN; provided expert testimony for Shuttleworth \& Ingersoll law firm. J. Brozek has nothing to disclose. D.E. Griffith served on an advisory committee, as a consultant, as a speaker and received research support from Insmed; served as a consultant for Johnson \& Johnson, Merck, and Spero. L. Guglielmetti has nothing to disclose. G.A. Huitt served on an advisory committee for Hill-Rom and Insmed. S.L. Knight has nothing to disclose. P. Leitman served as the president of NTM Info \& Research, Inc, during which time the organization received support from Insmed, Grifols, BeyondAir, Aradigm, Spero Therapeutics, Johnson \& Johnson, Hill-Rom, International Biophysics, Electromed, RespirTech, Maxor Specialty Pharmacy, PantherX, and Kroger Specialty Pharmacy. T.K. Marras served as a consultant and received research support from Insmed; served as a speaker for AstraZeneca and Novartis; served as a consultant for Horizon, Spero, and RedHill Biopharma. K.N. Olivier received research support from AIT Therapeutics, Insmed, and Matinas Biopharma. M. Santin received personal fees from DiaSorin SPA and Vircell SL. J.E. Stout has nothing to disclose. E. Tortoli has nothing to disclose. J. van Ingen served on an advisory committee and as a consultant for Insmed; served on advisory committees for Janssen Pharmaceuticals and Spero. D. Wagner served as a speaker for Cepheid GmbH; received research support and travel expenses from Insmed. K.L. Winthrop served on an advisory committee for Insmed, Johnson and Johnson, Paratek, Redhill Biopharma, and Spero; served as a consultant for Bayer Healthcare, Bristol-Myers Squibb, Horizon, Lilly, Pfizer, and RedHill Biopharma; received research support from Bristol-Myers Squibb, Cellestis, and Insmed; served on data safety and monitoring boards for Abbvie, Biomarin, Gilead, Roche, and UCB.

\section{References}

1 Schünemann HJ, Jaeschke R, Cook DJ, et al. An official ATS statement: grading the quality of evidence and strength of recommendations in ATS guidelines and recommendations. Am J Respir Crit Care Med 2006; 174: 605-614.

2 Guyatt G, Oxman AD, Akl EA, et al. GRADE guidelines: 1. Introduction-GRADE evidence profiles and summary of findings tables. J Clin Epidemiol 2011; 64: 383-394.

3 Andrews JC, Schünemann HJ, Oxman AD, et al. GRADE guidelines: 15. Going from evidence to recommendation-determinants of a recommendation's direction and strength. J Clin Epidemiol 2013; 66: $726-735$.

4 Griffith DE, Aksamit T, Brown-Elliott BA, et al. An official ATS/IDSA statement: diagnosis, treatment, and prevention of nontuberculous mycobacterial diseases. Am J Respir Crit Care Med 2007; 175: 367-416.

5 Tsukamura M. Diagnosis of disease caused by Mycobacterium avium complex. Chest 1991; 99: 667-669.

6 Koh WJ, Chang B, Ko Y, et al. Clinical significance of a single isolation of pathogenic nontuberculous mycobacteria from sputum specimens. Diagn Microbiol Infect Dis 2013; 75: 225-226.

7 Lee MR, Yang CY, Shu CC, et al. Factors associated with subsequent nontuberculous mycobacterial lung disease in patients with a single sputum isolate on initial examination. Clin Microbiol Infect 2015; 21: 250.

8 van Ingen J, Bendien SA, de Lange WC, et al. Clinical relevance of non-tuberculous mycobacteria isolated in the Nijmegen-Arnhem region, The Netherlands. Thorax 2009; 64: 502-506.

9 Jankovic M, Sabol I, Zmak L, et al. Microbiological criteria in non-tuberculous mycobacteria pulmonary disease: a tool for diagnosis and epidemiology. Int J Tuberc Lung Dis 2016; 20: 934-940.

10 van Ingen J, Boeree MJ, van Soolingen D, et al. Are phylogenetic position, virulence, drug susceptibility and in vivo response to treatment in mycobacteria interrelated? Infect Genet Evol 2012; 12: 832-837.

11 Koh WJ, Moon SM, Kim SY, et al. Outcomes of Mycobacterium avium complex lung disease based on clinical phenotype. Eur Respir J 2017; 50: 1602503.

12 Koh WJ, Jeong BH, Kim SY, et al. Mycobacterial characteristics and treatment outcomes in Mycobacterium abscessus lung disease. Clin Infect Dis 2017; 64: 309-316.

13 Wallace RJ Jr, Zhang Y, Brown-Elliott BA, et al. Repeat positive cultures in Mycobacterium intracellulare lung disease after macrolide therapy represent new infections in patients with nodular bronchiectasis. J Infect Dis 2002; 186: 266-273.

14 CLSI. Susceptibility testing of mycobacteria, Nocardia spp, and other aerobic actinomyces. 3rd Edn. Vol. M24. Wayne, PA, Clinical and Laboratory Standards Institute, 2018.

15 CLSI. Performance standards for susceptibility testing of mycobacteria, Nocardia spp, and other aerobic actinomyces. 1st Edn. Vol. M62. Wayne, PA, Clinical and Laboratory Standards Institute, 2018.

16 Griffith DE, Brown-Elliott BA, Langsjoen B, et al. Clinical and molecular analysis of macrolide resistance in Mycobacterium avium complex lung disease. Am J Respir Crit Care Med 2006; 174: 928-934.

17 Moon SM, Park HY, Kim SY, et al. Clinical characteristics, treatment outcomes, and resistance mutations associated with macrolide-resistant Mycobacterium avium complex lung disease. Antimicrob Agents Chemother 2016; 60: 6758-6765. 
Morimoto K, Namkoong H, Hasegawa N, et al. Macrolide-resistant Mycobacterium avium complex lung disease: analysis of 102 consecutive cases. Ann Am Thorac Soc 2016; 13: 1904-1911.

Olivier KN, Griffith DE, Eagle G, et al. Randomized trial of liposomal amikacin for inhalation in nontuberculous mycobacterial lung disease. Am J Respir Crit Care Med 2017; 195: 814-823.

Griffith DE, Eagle G, Thomson R, et al. Amikacin liposome inhalation suspension for treatment-refractory lung disease caused by Mycobacterium avium complex (CONVERT). A prospective, open-label, randomized study. Am J Respir Crit Care Med 2018; 198: 1559-1569.

Miwa S, Shirai M, Toyoshima M, et al. Efficacy of clarithromycin and ethambutol for Mycobacterium avium complex pulmonary disease: a preliminary study. Ann Am Thorac Soc 2014; 11: 23-29.

Wallace RJ Jr, Brown-Elliott BA, McNulty S, et al. Macrolide/azalide therapy for nodular/bronchiectatic Mycobacterium avium complex lung disease. Chest 2014; 146: 276-282.

Jeong BH, Jeon K, Park HY, et al. Intermittent antibiotic therapy for nodular bronchiectatic Mycobacterium avium complex lung disease. Am J Respir Crit Care Med 2015; 191: 96-103.

Harris GD, Johanson WG, Nicholson DP. Response to chemotherapy of pulmonary infection due to Mycobacterium kansasii. Am Rev Respir Dis 1975; 112: 31-36.

Shitrit D, Baum GL, Priess R, et al. Pulmonary Mycobacterium kansasii infection in Israel, 1999-2004: clinical features, drug susceptibility, and outcome. Chest 2006; 129: 771-776.

Griffith DE, Brown-Elliott BA, Wallace RJ Jr. Thrice-weekly clarithromycin-containing regimen for treatment of Mycobacterium kansasii lung disease: results of a preliminary study. Clin Infect Dis 2003; 37: 1178-1182.

Sauret J, Hernández-Flix S, Castro E, et al. Treatment of pulmonary disease caused by Mycobacterium kansasii results of 18 vs 12 months' chemotherapy. Tuber Lung Dis 1995; 76: 104-108.

Santin M, Dorca J, Alcaide F, et al. Long-term relapses after 12-month treatment for Mycobacterium kansasii lung disease. Eur Respir J 2009; 33: 148-152.

Ahn CH, Lowell JR, Ahn SS, et al. Short-course chemotherapy for pulmonary disease caused by Mycobacterium kansasii. Am Rev Respir Dis 1983; 128: 1048-1050.

Pezzia W, Raleigh JW, Bailey MC, et al. Treatment of pulmonary disease due to Mycobacterium kansasii: recent experience with rifampin. Rev Infect Dis 1981; 3: 1035-1039.

Ahn CH, Lowell JR, Ahn SS, et al. Chemotherapy for pulmonary disease due to Mycobacterium kansasii: efficacies of some individual drugs. Rev Infect Dis 1981; 3: 1028-1034.

van Ingen J, Hoefsloot W, Mouton JW, et al. Synergistic activity of rifampicin and ethambutol against slow-growing nontuberculous mycobacteria is currently of questionable clinical significance. Int J Antimicrob Agents 2013; 42: 80-82.

Andrejak C, Veziris N, Lescure FX, et al. Camomy Trial: a prospective randomized clinical trial to compare six-months sputum conversion rate with a clarithromycin or moxifloxacin containing regimen in patients with a M. xenopi pulmonary infection: intermediate analysis. Am J Respir Crit Care Med 2016; 193: A3733.

Marras TK, Campitelli MA, Lu H, et al. Pulmonary nontuberculous mycobacteria-associated deaths, Ontario, Canada, 2001-2013. Emerg Infect Dis 2017; 23: 468-476.

Andréjak C, Lescure FX, Pukenyte E, et al. Mycobacterium xenopi pulmonary infections: a multicentric retrospective study of 136 cases in north-east France. Thorax 2009; 64: 291-296.

Jenkins PA, Campbell IA, Research Committee of The British Thoracic Society. Pulmonary disease caused by Mycobacterium xenopi in -negative patients: five year follow-up of patients receiving standardised treatment. Respir Med 2003; 97: 439-444.

Banks J, Hunter AM, Campbell IA, et al. Pulmonary infection with Mycobacterium xenopi: review of treatment and response. Thorax 1984; 39: 376-382.

Koh WJ, Jeon K, Lee NY, et al. Clinical significance of differentiation of Mycobacterium massiliense from Mycobacterium abscessus. Am J Respir Crit Care Med 2011; 183: 405-410.

Jeon K, Kwon OJ, Lee NY, et al. Antibiotic treatment of Mycobacterium abscessus lung disease: a retrospective analysis of 65 patients. Am J Respir Crit Care Med 2009; 180: 896-902.

Bastian S, Veziris N, Roux AL, et al. Assessment of clarithromycin susceptibility in strains belonging to the Mycobacterium abscessus group by erm(41) and rrl sequencing. Antimicrob Agents Chemother 2011; 55: 775-781.

Mougari F, Bouziane F, Crockett F, et al. Selection of resistance to clarithromycin in Mycobacterium abscessus subspecies. Antimicrob Agents Chemother 2017; 61: e00943-16.

Mougari F, Amarsy R, Veziris N, et al. Standardized interpretation of antibiotic susceptibility testing an resistance genotyping for Mycobacterium abscessus with regard to subspecies and erm41 sequevar. J Antimicrob Chemother 2016; 71: 2208-2212.

Mitchell JD, Bishop A, Cafaro A, et al. Anatomic lung resection for nontuberculous mycobacterial disease. Ann Thorac Surg 2008; 85: 1887-1892.

Marras TK, Mendelson D, Marchand-Austin A, et al. Pulmonary nontuberculous mycobacterial disease, Ontario, Canada, 1998-2010. Emerg Infect Dis 2013; 19: 1889-1891.

Adjemian J, Olivier KN, Seitz AE, et al. Prevalence of nontuberculous mycobacterial lung disease in US Medicare beneficiaries. Am J Respir Crit Care Med 2012; 185: 881-886.

Prevots DR, Marras TK. Epidemiology of human pulmonary infection with nontuberculous mycobacteria: a review. Clin Chest Med 2015; 36: 13-34.

Henkle E, Hedberg K, Schafer S, et al. Population-based incidence of pulmonary nontuberculous mycobacterial disease in Oregon 2007 to 2012. Ann Am Thorac Soc 2015; 12: 642-647.

van Ingen J, Hoefsloot W, Dekhuijzen PN, et al. The changing pattern of clinical Mycobacterium avium isolation in the Netherlands. Int J Tuberc Lung Dis 2010; 14: 1176-1180.

van Ingen J, Turenne CY, Tortoli E, et al. A definition of the Mycobacterium avium complex for taxonomical and clinical purposes, a review. Int J Syst Evol Microbiol 2018; 68: 3666-3677.

Guyatt GH, Oxman AD, Kunz R, et al. GRADE guidelines: 2. Framing the question and deciding on important outcomes. J Clin Epidemiol 2011; 64: 395-400.

Sugihara E, Hirota N, Niizeki T, et al. Usefulness of bronchial lavage for the diagnosis of pulmonary disease caused by Mycobacterium avium-intracellulare complex (MAC) infection. J Infect Chemother 2003; 9: $328-332$. 
Tanaka E, Amitani R, Niimi A, et al. Yield of computed tomography and bronchoscopy for the diagnosis of Mycobacterium avium complex pulmonary disease. Am J Respir Crit Care Med 1997; 155: 2041-2046.

53 Huang JH, Kao PN, Adi V, et al. Mycobacterium avium-intracellulare pulmonary infection in HIV-negative patients without preexisting lung disease: diagnostic and management limitations. Chest 1999; 115: 1033-1040.

54 Watanuki Y, Odagiri S, Suzuki K, et al. Usefulness of bronchoscopy for the diagnosis of atypical pulmonary mycobacteriosis. Kansenshogaku Zasshi 1999; 73: 728-733.

55 Ikedo Y. The significance of bronchoscopy for the diagnosis of Mycobacterium avium complex (MAC) pulmonary disease. Kurume Med J 2001; 48: 15-19.

56 Peres RL, Maciel EL, Morais CG, et al. Comparison of two concentrations of NALC-NaOH for decontamination of sputum for mycobacterial culture. Int J Tuberc Lung Dis 2009; 13: 1572-1575.

57 Cruciani M, Scarparo C, Malena M, et al. Metaanalysis of BACTEC MGIT 960 and BACTEC 460 TB, with or without solid media, for detection of mycobacteria. J Clin Microbiol 2004; 42: 2321-2325.

58 Chew WK, Lasaitis RM, Schio FA, et al. Clinical evaluation of the Mycobacteria Growth Indicator Tube (MGIT) compared with radiometric (Bactec) and solid media for isolation of Mycobacterium species. J Med Microbiol 1998; 47: 821-827.

59 Idigoras P, Beristain X, Iturzaeta A, et al. Comparison of the automated nonradiometric Bactec MGIT 960 system with Löwenstein-Jensen, Coletsos, and Middlebrook 7H11 solid media for recovery of mycobacteria. Eur J Clin Microbiol Infect Dis 2000; 19: 350-354.

60 Sorlozano A, Soria I, Roman J, et al. Comparative evaluation of three culture methods for the isolation of mycobacteria from clinical samples. J Microbiol Biotechnol 2009; 19: 1259-1264.

61 Rivera AB, Tupasi TE, Grimaldo ER, et al. Rapid and improved recovery rate of Mycobacterium tuberculosis in mycobacteria growth indicator tube combined with solid Löwenstein Jensen medium. Int J Tuberc Lung Dis 1997; 1: 454-459.

62 Alcaide F, Benítez MA, Escribà JM, et al. Evaluation of the BACTEC MGIT 960 and the MB/BacT systems for recovery of mycobacteria from clinical specimens and for species identification by DNA AccuProbe. $J$ Clin Microbiol 2000; 38: 398-401.

63 Lu D, Heeren B, Dunne WM. Comparison of the automated mycobacteria growth indicator tube system (BACTEC 960/MGIT) with Löwenstein-Jensen medium for recovery of mycobacteria from clinical specimens. Am J Clin Pathol 2002; 118: 542-545.

64 Lee JJ, Suo J, Lin CB, et al. Comparative evaluation of the BACTEC MGIT 960 system with solid medium for isolation of mycobacteria. Int J Tuberc Lung Dis 2003; 7: 569-574.

65 Hillemann D, Richter E, Rüsch-Gerdes S. Use of the BACTEC mycobacteria growth indicator tube 960 automated system for recovery of mycobacteria from 9,558 extrapulmonary specimens, including urine samples. $J$ Clin Microbiol 2006; 44: 4014-4017.

66 CLSI. Laboratory detection and identification of mycobacteria. 2nd Edn. Vol. M48. Wayne, PA, Clinical and Laboratory Standards Institute, 2018.

67 Alfa MJ, Manickam K, Sepehri S, et al. Evaluation of BacT/Alert 3D automated unit for detection of nontuberculous mycobacteria requiring incubation at 30 degrees C for optimal growth. J Clin Microbiol 2011; 49: 2691-2693.

68 Peter-Getzlaff S, Lüthy J, Voit A, et al. Detection and identification of Mycobacterium spp. in clinical specimens by combining the Roche Cobas amplicor Mycobacterium tuberculosis assay with Mycobacterium genus detection and nucleic acid sequencing. J Clin Microbiol 2010; 48: 3943-3948.

69 Deggim-Messmer V, Bloemberg GV, Ritter C, et al. Diagnostic molecular mycobacteriology in regions with low tuberculosis endemicity: combining realtime PCR assays for detection of multiple mycobacterial pathogens with line probe assays for identification of resistance mutations. EBioMedicine 2016; 9: 228-237. van Ingen J, de Zwaan R, Enaimi M, et al. Re-analysis of 178 previously unidentifiable Mycobacterium isolates in the Netherlands in 1999-2007. Clin Microbiol Infect 2010; 16: 1470-1474.

71 Tortoli E, Pecorari M, Fabio G, et al. Commercial DNA probes for mycobacteria incorrectly identify a number of less frequently encountered species. J Clin Microbiol 2010; 48: 307-310.

72 McNabb A, Eisler D, Adie K, et al. Assessment of partial sequencing of the 65-kilodalton heat shock protein gene (hsp65) for routine identification of Mycobacterium species isolated from clinical sources. J Clin Microbiol 2004; 42: 3000-3011.

73 Adékambi T, Colson P, Drancourt M. rpoB-based identification of nonpigmented and late-pigmenting rapidly growing mycobacteria. J Clin Microbiol 2003; 41: 5699-5708.

74 de Zwaan R, van Ingen J, van Soolingen D. Utility of rpoB gene sequencing for identification of nontuberculous mycobacteria in the Netherlands. J Clin Microbiol 2014; 52: 2544-2551.

75 Roth A, Fischer M, Hamid ME, et al. Differentiation of phylogenetically related slowly growing mycobacteria based on 16S-23S rRNA gene internal transcribed spacer sequences. J Clin Microbiol 1998; 36: 139-147.

76 van Ingen J, Hoefsloot W, Buijtels PC, et al. Characterization of a novel variant of Mycobacterium chimaera. J Med Microbiol 2012; 61: 1234-1239.

77 Macheras E, Roux AL, Bastian S, et al. Multilocus sequence analysis and rpoB sequencing of Mycobacterium abscessus (sensu lato) strains. J Clin Microbiol 2011; 49: 491-499.

78 Zelazny AM, Root JM, Shea YR, et al. Cohort study of molecular identification and typing of Mycobacterium abscessus, Mycobacterium massiliense, and Mycobacterium bolletii. J Clin Microbiol 2009; 47: $1985-1995$.

79 Alcaide F, Amlerová J, Bou G, et al. How to: identify non-tuberculous Mycobacterium species using MALDI-TOF mass spectrometry. Clin Microbiol Infect 2018; 24: 599-603.

80 Buchan BW, Riebe KM, Timke M, et al. Comparison of MALDI-TOF MS with HPLC and nucleic acid sequencing for the identification of Mycobacterium species in cultures using solid medium and broth. Am J Clin Pathol 2014; 141: 25-34.

81 Leyer C, Gregorowicz G, Mougari F, et al. Comparison of Saramis 4.12 and IVD 3.0 Vitek MS matrix-assisted laser desorption ionization-time of flight mass spectrometry for identification of mycobacteria from solid and liquid culture media. J Clin Microbiol 2017; 55: 2045-2054.

82 van Eck K, Faro D, Wattenberg M, et al. Matrix-assisted laser desorption ionization-time of flight mass spectrometry fails to identify nontuberculous mycobacteria from primary cultures of respiratory samples. $\mathrm{J} C$ lin Microbiol 2016; 54: 1915-1917. 
114 Wallace

115 Ahn $\mathrm{CH}$, Wallace RJ Jr, Steele LC, et al. Sulfonamide-containing regimens for disease caused by rifampin-resistant Mycobacterium kansasii. Am Rev Respir Dis 1987; 135: 10-16.

Wallace RJ Jr, Brown BA, Griffith DE, et al. Clarithromycin regimens for pulmonary Mycobacterium avium complex: the first 50 patients. Am J Respir Crit Care Med 1996; 153: 1766-1772.

Tanaka E, Kimoto T, Tsuyuguchi K, et al. Effect of clarithromycin regimen for Mycobacterium avium complex pulmonary disease. Am J Respir Crit Care Med 1999; 160: 866-872.

Meier A, Heifets L, Wallace RJ Jr, et al. Molecular mechanisms of clarithromycin resistance in Mycobacterium avium: observation of multiple $23 \mathrm{~S}$ rDNA mutations in a clonal population. J Infect Dis 1996; 174: 354-360

Meier A, Kirschner P, Springer B, et al. Identification of mutations in 23S rRNA gene of clarithromycin-resistant Mycobacterium intracellulare. Antimicrob Agents Chemother 1994; 38: 381-384.

(1) BA, lakhiaeva E, Griffith DE, et al. In vitro activity of amikaci avium complex with proposed MIC breakpoints and finding of a $16 \mathrm{~S}$ rRNA gene mutation in treated isolates. Clin Microbiol 2013; 51: 3389-3394.

(CLSI). CaLSI. Susceptibility testing of mycobacteria, nocardiae, and other aerobic actinomycetes: approved standard-second edition. CLSI document M24-A2. Wayne, Pennsylvania, Clinical Laboratory Standards Institute, 2011. Jarand J, Levin A, Zhang L, et al. Clinical and microbiologic outcomes in patients receiving treatment for Mycobacterium abscessus pulmonary disease. Clin Infect Dis 2011; 52: 565-571. susceptibility testing. J Microbiol Immunol Infect 2010; 43: 401-406.

van Ingen J, Totten SE, Helstrom NK, et al. In vitro synergy between clofazimine and amikacin in treatment of nontuberculous mycobacterial disease. Antimicrob Agents Chemother 2012; 56: 6324-6327.

, Wattenberg M, et al. Clofa Mycobacterium avium type strains exposed to amikacin and clarithromycin. Antimicrob Agents Chemother 2016; 60: 1097-1105.

Nash KA, Brown-Elliott BA, Wallace RJ Jr. A novel gene, erm(41), confers inducible macrolide resistance to clinical isolates of Mycobacterium abscessus but is absent from Mycobacterium chelonae. Antimicrob Agents Chemother 2009; 53: 1367-1376.

Wallace RJ Jr, Meier A, Brown BA, et al. Genetic basis for clarithromycin resistance among isolates of Mycobacterium chelonae and Mycobacterium abscessus. Antimicrob Agents Chemother 1996; 40: 1676-1681.

Ito Y, Hirai T, Maekawa K, et al. Predictors of 5-year mortality in pulmonary Mycobacterium avium-intracellulare complex disease. Int J Tuberc Lung Dis 2012; 16: 408-414.

Zoumot Z, Boutou AK, Gill SS, et al. Mycobacterium avium complex infection in non-cystic fibrosis bronchiectasis. Respirology 2014; 19: 714-722.

Hwang JA, Kim S, Jo KW, et al. Natural history of Mycobacterium avium com plex lung disease in untreated patients with stable course. Eur Respir J 2017; 49: 1600537.

Hunter AM, Campbell IA, Jenkins PA, et al. Treatment of pulmonary infections caused by mycobacteria of the Mycobacterium avium-intracellulare complex. Thorax 1981; 36: 326-329.

G, Lee KS, Moon JW, et al. Nodular bronchiectatic Mycobacterium avium complex pulmonary disease. Natural course on serial computed tomographic scans. Ann Am Thorac Soc 2013; 10: 299-306. non-tuberculous mycobacteria: a retrospective cohort study. Respir Med 2015; 109: 137-145.

Andréjak C, Lescure FX, Douadi Y, et al. Non-tuberculous mycobacteria pulmonary infection: management and follow-up of 31 infected patients. J Infect 2007; 55: 34-40.

Gochi M, Takayanagi N, Kanauchi T, et al. Retrospective study of the predictors of mortality and radiographic deterioration in 782 patients with nodular/bronchiectatic Mycobacterium avium complex lung disease. BMJ Open 2015; 5: e008058.

Prevots DR, Shaw PA, Strickland D, et al. Nontuberculous mycobacterial lung disease prevalence at four integrated health care delivery systems. Am J Respir Crit Care Med 2010; 182: 970-976.

Mycobacterium avium complex lung disease. Am J Respir Crit Care Med 2012; 185: 575-583.

Rawson TM, Abbara A, Kranzer $\mathrm{K}$, et al. Factors which influence treatment initiation for pulmonary non-tuberculous Mycobacterium infection in HIV negative patients; a multicentre observational study. Respir Med 2016; 120: 101-108.

Respir Med 2011; 105: 1718-1725.

Hong JY, Lee SA, Kim SY, et al. Factors associated with quality of life measured by EQ-5D in patients with nontuberculous mycobacterial pulmonary disease. Qual Life Res 2014; 23: 2735-2741.

group lung infection: a prospective cohort study. Ann Am Thorac Soc 2016; 13: 40-48.

Mycobacterium avium complex pulmonary disease. Int J Tuberc Lung Dis 2012; 16: 660-664.

Brown-Elliott BA, Nash KA, Wallace RJ Jr. Antimicrobial susceptibility testing, drug resistance mechanisms, and therapy of infections with nontuberculous mycobacteria. Clin Microbiol Rev 2012; 25: 545-582. nontuberculous mycobacteria. Drug Resist Updat 2012; 15: 149-161.

Kobashi Y, Yoshida K, Miyashita N, et al. Relationship between clinical efficacy of treatment of pulmonary Mycobacterium avium complex disease and drug-sensitivity testing of Mycobacterium avium complex isolates. $J$ Infect Chemother 2006; 12: 195-202.

CA, Dube MP, et al. Clarithromycin therapy for bacteremic Mycobacterium avium complex disease: a randomized, double-blind, dose-ranging study in patients with AIDS. AIDS Clinical Trials Group Protocol 157 Study Team. Ann Intern Med 1994; 121: 905-911.

RJ Jr, Dunbar D, Brown BA, et al. Rifampin-resistant Mycobacterium kansasii. Clin Infect Dis 1994; 18 
116 Sison JP, Yao Y, Kemper CA, et al. Treatment of Mycobacterium avium complex infection: do the results of in vitro susceptibility tests predict therapeutic outcome in humans? J Infect Dis 1996; 173: 677-683.

117 Wallace RJ Jr, Brown BA, Griffith DE, et al. Initial clarithromycin monotherapy for Mycobacterium avium-intracellulare complex lung disease. Am J Respir Crit Care Med 1994; 149: 1335-1341.

118 Kobashi Y, Abe M, Mouri K, et al. Relationship between clinical efficacy for pulmonary MAC and drug-sensitivity test for isolated MAC in a recent 6-year period. J Infect Chemother 2012; 18: 436-443.

119 Research Committee of the British Thoracic Society. First randomised trial of treatments for pulmonary disease caused by $M$. avium intracellulare, M. malmoense, and M. xenopi in HIV negative patients: rifampicin, ethambutol and isoniazid versus rifampicin and ethambutol. Thorax 2001; 56: 167-172.

120 Prammananan T, Sander P, Brown BA, et al. A single 16S ribosomal RNA substitution is responsible for resistance to amikacin and other 2-deoxystreptamine aminoglycosides in Mycobacterium abscessus and Mycobacterium chelonae. J Infect Dis 1998; 177: 1573-1581.

121 Kobashi Y, Matsushima T, Oka M. A double-blind randomized study of aminoglycoside infusion with combined therapy for pulmonary Mycobacterium avium complex disease. Respir Med 2007; 101: 130-138.

122 Smith MJ, Citron KM. Clinical review of pulmonary disease caused by Mycobacterium xenopi. Thorax 1983; 38: 373-377.

123 van Ingen J, Boeree MJ, de Lange WC, et al. Mycobacterium xenopi clinical relevance and determinants, the Netherlands. Emerg Infect Dis 2008; 14: 385-389.

124 Choi H, Kim SY, Lee H, et al. Clinical characteristics and treatment outcomes of patients with macrolide-resistant Mycobacterium massiliense lung disease. Antimicrob Agents Chemother 2017; 61: e02189-16.

125 Maurer FP, Rüegger V, Ritter C, et al. Acquisition of clarithromycin resistance mutations in the 23S rRNA gene of Mycobacterium abscessus in the presence of inducible erm(41). J Antimicrob Chemother 2012; 67: 2606-2611.

126 Dautzenberg B, Truffot C, Legris S, et al. Activity of clarithromycin against Mycobacterium avium infection in patients with the acquired immune deficiency syndrome: a controlled clinical trial. Am Rev Respir Dis 1991; 144: 564-569.

127 Pierce M, Crampton S, Henry D, et al. A randomized trial of clarithromycin as prophylaxis against disseminated Mycobacterium avium complex infection in patients with advanced acquired immunodeficiency syndrome. $N$ Engl J Med 1996; 335: 384-391.

128 Havlir DV, Dubé MP, Sattler FR, et al. Prophylaxis against disseminated Mycobacterium avium complex with weekly azithromycin, daily rifabutin, or both. California Collaborative Treatment Group. N Engl J Med 1996; 335: 392-398.

129 Shafran SD, Singer J, Zarowny DP, et al. A comparison of two regimens for the treatment of Mycobacterium avium complex bacteremia in AIDS: rifabutin, ethambutol, and clarithromycin versus rifampin, ethambutol, clofazimine, and ciprofloxacin. Canadian HIV Trials Network Protocol 010 Study Group. N Engl J Med 1996; 335: 377-383.

130 Benson CA, Williams PL, Currier JS, et al. A prospective, randomized trial examining the efficacy and safety of clarithromycin in combination with ethambutol, rifabutin, or both for the treatment of disseminated Mycobacterium avium complex disease in persons with acquired immunodeficiency syndrome. Clin Infect Dis 2003; 37: 1234-1243.

131 Jenkins PA, Campbell IA, Banks J, et al. Clarithromycin vs ciprofloxacin as adjuncts to rifampicin and ethambutol in treating opportunist mycobacterial lung diseases and an assessment of Mycobacterium vaccae immunotherapy. Thorax 2008; 63: 627-634.

132 Fujita M, Kajiki A, Tao Y, et al. The clinical efficacy and safety of a fluoroquinolonecontaining regimen for pulmonary MAC disease. J Infect Chemother 2012; 18: 146-151.

133 Pasipanodya JG, Ogbonna D, Deshpande D, et al. Metaanalyses and the evidence base for microbial outcomes in the treatment of pulmonary Mycobacterium avium-intracellulare complex disease. J Antimicrob Chemother 2017; 72: i3-i19.

134 Diel R, Nienhaus A, Ringshausen FC, et al. Microbiologic outcome of interventions against Mycobacterium avium complex pulmonary disease: a systematic review. Chest 2018; 153: 888-921.

135 Jarand J, Davis JP, Cowie RL, et al. Long-term follow-up of Mycobacterium avium complex lung disease in patients treated with regimens including clofazimine and/or rifampin. Chest 2016; 149: 1285-1293.

136 Kadota JI, Kurashima A, Suzuki K. The clinical efficacy of a clarithromycin-based regimen for Mycobacterium avium complex disease: a nationwide post-marketing study. I Infect Chemother 2017; 23: 293-300.

137 Field SK, Fisher D, Cowie RL. Mycobacterium avium complex pulmonary disease in patients without HIV infection. Chest 2004; 126: 566-581.

138 Griffith DE, Brown BA, Girard WM, et al. Azithromycin activity against Mycobacterium avium complex lung disease in patients who were not infected with human immunodeficiency virus. Clin Infect Dis 1996; 23: 983-989.

139 Griffith DE, Brown BA, Cegielski P, et al. Early results (at 6 months) with intermittent clarithromycin-including regimens for lung disease due to Mycobacterium avium complex. Clin Infect Dis 2000; 30: 288-292.

140 Griffith DE, Brown BA, Girard WM, et al. Azithromycin-containing regimens for treatment of Mycobacterium avium complex lung disease. Clin Infect Dis 2001; 32: 1547-1553.

141 Field SK, Cowie RL. Treatment of Mycobacterium avium-intracellulare complex lung disease with a macrolide, ethambutol, and clofazimine. Chest 2003; 124: 1482-1486.

142 Rubinstein E. Comparative safety of the different macrolides. Int J Antimicrob Agents 2001; 18: Suppl. 1, S71-S76.

143 Griffith DE, Brown BA, Girard WM, et al. Adverse events associated with high-dose rifabutin in macrolide-containing regimens for the treatment of Mycobacterium avium complex lung disease. Clin Infect Dis 1995; 21: 594-598.

144 Mitnick CD, McGee B, Peloquin CA. Tuberculosis pharmacotherapy: strategies to optimize patient care. Expert Opin Pharmacother 2009; 10: 381-401.

145 van Ingen $\mathrm{J}$, Egelund $\mathrm{EF}$, Levin $\mathrm{A}$, et al. The pharmacokinetics and pharmacodynamics of pulmonary Mycobacterium avium complex disease treatment. Am J Respir Crit Care Med 2012; 186: 559-565. 
Yeates RA, Laufen H, Zimmermann T. Interaction between midazolam and clarithromycin: comparison with azithromycin. Int I Clin Pharmacol Ther 1996; 34: 400-405.

Schembri S, Williamson PA, Short PM, et al. Cardiovascular events after clarithromycin use in lower respiratory tract infections: analysis of two prospective cohort studies. BMJ 2013; 346: f1235.

Gluud C, Als-Nielsen B, Damgaard M, et al. Clarithromycin for 2 weeks for stable coronary heart disease: 6-year follow-up of the CLARICOR randomized trial and updated meta-analysis of antibiotics for coronary heart disease. Cardiology 2008; 111: 280-287.

Hansen MP, Scott AM, McCullough A, et al. Adverse events in people taking macrolide antibiotics versus placebo for any indication. Cochrane Database Syst Rev 2019; 1: CD011825.

Brown BA, Griffith DE, Girard W, et al. Relationship of adverse events to serum drug levels in patients receiving high-dose azithromycin for mycobacterial lung disease. Clin Infect Dis 1997; 24: 958-964.

Wallace RJ Jr, Brown BA, Griffith DE. Drug intolerance to high-dose clarithromycin among elderly patients. Diagn Microbiol Infect Dis 1993; 16: 215-221.

Medical Section of the American Lung Association. Diagnosis and treatment of disease caused by nontuberculous mycobacteria. This official statement of the American Thoracic Society was approved by the Board of Directors, March 1997. Am J Respir Crit Care Med 1997; 156: S1-S25.

Zweijpfenning S, Kops S, Magis-Escurra C, et al. Treatment and outcome of non-tuberculous mycobacterial pulmonary disease in a predominantly fibro-cavitary disease cohort. Respir Med 2017; 131: 220-224.

Peloquin CA, Berning SE, Nitta AT, et al. Aminoglycoside toxicity: daily versus thrice-weekly dosing for treatment of mycobacterial diseases. Clin Infect Dis 2004; 38: 1538-1544.

Davis KK, Kao PN, Jacobs SS, et al. Aerosolized amikacin for treatment of pulmonary Mycobacterium avium infections: an observational case series. BMC Pulm Med 2007; 7: 2

Safdar A. Aerosolized amikacin in patients with difficult-to-treat pulmonary nontuberculous mycobacteriosis. Eur J Clin Microbiol Infect Dis 2012; 31: 1883-1887.

Olivier KN, Shaw PA, Glaser TS, et al. Inhaled amikacin for treatment of refractory pulmonary nontuberculous mycobacterial disease. Ann Am Thorac Soc 2014; 11: 30-35.

Jhun BW, Yang B, Moon SM, et al. Amikacin inhalation as salvage therapy for refractory nontuberculous mycobacterial lung disease. Antimicrob Agents Chemother 2018; 62: e00011-18.

Yagi K, Ishii M, Namkoong $\mathrm{H}$, et al. The efficacy, safety, and feasibility of inhaled amikacin for the treatment of difficult-to-treat non-tuberculous mycobacterial lung diseases. BMC Infect Dis 2017; 17: 558.

Daley CL, Glassroth J. Treatment of pulmonary nontuberculous mycobacterial infections: many questions remain. Ann Am Thorac Soc 2014; 11: 96-97.

Gordin FM, Sullam PM, Shafran SD, et al. A randomized, placebo-controlled study of rifabutin added to a regimen of clarithromycin and ethambutol for treatment of disseminated infection with Mycobacterium avium complex. Clin Infect Dis 1999; 28: 1080-1085.

Cohn DL, Catlin BJ, Peterson KL, et al. A 62-dose, 6-month therapy for pulmonary and extrapulmonary tuberculosis: a twice-weekly, directly observed, and cost-effective regimen. Ann Intern Med 1990; 112: 407-415.

Lam PK, Griffith DE, Aksamit TR, et al. Factors related to response to intermittent treatment of Mycobacterium avium complex lung disease. Am J Respir Crit Care Med 2006; 173: 1283-1289.

Griffith DE, Brown BA, Murphy DT, et al. Initial (6-month) results of three-times-weekly azithromycin in treatment regimens for Mycobacterium avium complex lung disease in human immunodeficiency virus-negative patients. J Infect Dis 1998; 178: 121-126.

Griffith DE, Brown-Elliott BA, Shepherd S, et al. Ethambutol ocular toxicity in treatment regimens for Mycobacterium avium complex lung disease. Am J Respir Crit Care Med 2005; 172: 250-253.

Kwak N, Park J, Kim E, et al. Treatment outcomes of Mycobacterium avium complex lung disease: a systematic review and meta-analysis. Clin Infect Dis 2017; 65: 1077-1084.

Koh WJ, Jeong BH, Jeon $\mathrm{K}$, et al. Response to switch from intermittent therapy to daily therapy for refractory nodular bronchiectatic Mycobacterium avium complex lung disease. Antimicrob Agents Chemother 2015; 59: 4994-4996.

Jhun BW, Moon SM, Kim SY, et al. Intermittent antibiotic therapy for recurrent nodular bronchiectatic Mycobacterium avium complex lung disease. Antimicrob Agents Chemother 2018; 62: e01787-19.

Griffith DE, Adjemian J, Brown-Elliott BA, et al. Semiquantitative culture analysis during therapy for Mycobacterium avium complex lung disease. Am J Respir Crit Care Med 2015; 192: 754-760.

van Ingen J, Aksamit T, Andrejak C, et al. Treatment outcome definitions in nontuberculous mycobacterial pulmonary disease: an NTM-NET consensus statement. Eur Respir J 2018; 51: 1800170.

Buhler VB, Pollak A. Human infection with atypical acid-fast organisms; report of two cases with pathologic findings. Am J Clin Pathol 1953; 23: 363-374.

Jenkins D, Bahar D, Chofnas I. Pulmonary disease due to atypical mycobacteria; current concepts. Transactions 19th Conference on Chemotherapy of Tuberculosis. 1960; pp. 224-231.

Research Committee, British Thoracic Society. Mycobacterium kansasii pulmonary infection: a prospective study of the results of nine months of treatment with rifampicin and ethambutol. Thorax 1994; 49: 442-445.

Alcaide F, Calatayud L, Santín M, et al. Comparative in vitro activities of linezolid, telithromycin, clarithromycin, levofloxacin, moxifloxacin, and four conventional antimycobacterial drugs against Mycobacterium kansasii. Antimicrob Agents Chemother 2004; 48: 4562-4565.

Guna R, Muñoz C, Domínguez V, et al. In vitro activity of linezolid, clarithromycin and moxifloxacin against clinical isolates of Mycobacterium kansasii. J Antimicrob Chemother 2005; 55: 950-953.

Brown BA, Wallace RJ Jr, Onyi GO. Activities of clarithromycin against eight slowly growing species of nontuberculous mycobacteria, determined by using a broth microdilution MIC system. Antimicrob Agents Chemother 1992; 36: 1987-1990.

Bakula Z, Modrzejewska M, Pennings L, et al. Drug susceptibility profiling and genetic determinants of drug resistance in Mycobacterium kansasii. Antimicrob Agents Chemother 2018; 62: e01788-17.

Philley JV, Griffith DE. Treatment of slowly growing mycobacteria. Clin Chest Med 2015; 36: 79-90

Hornick DB, Dayton CS, Bedell GN, et al. Nontuberculous mycobacterial lung disease: substantiation of a less aggressive approach. Chest 1988; 93: 550-555. 
180 Hombach M, Somoskövi A, Hömke R, et al. Drug susceptibility distributions in slowly growing non-tuberculous mycobacteria using MGIT 960 TB eXiST. Int J Med Microbiol 2013; 303: 270-276.

181 Srivastava S, Pasipanodya J, Sherman CM, et al. Rapid drug tolerance and dramatic sterilizing effect of moxifloxacin monotherapy in a novel hollow-fiber model of intracellular Mycobacterium kansasii disease. Antimicrob Agents Chemother 2015; 59: 2273-2279.

182 Jenkins PA, Banks J, Campbell IA, et al. Mycobacterium kansasii pulmonary infection: a prospective study of the results of nine months of treatment with rifampicin and ethambutol. Thorax 1994; 49: 442-445.

183 Shu CC, Lee CH, Hsu CL, et al. Clinical characteristics and prognosis of nontuberculous mycobacterial lung disease with different radiographic patterns. Lung 2011; 189: 467-474.

184 Diel R, Ringshausen F, Richter E, et al. Microbiological and clinical outcomes of treating non-Mycobacterium avium complex nontuberculous mycobacterial pulmonary disease: a systematic review and meta-analysis. Chest 2017; 152: 120-142.

185 Varadi RG, Marras TK. Pulmonary Mycobacterium xenopi infection in non-HIV-infected patients: a systematic review. Int J Tuberc Lung Dis 2009; 13: 1210-1218.

186 Andréjak C, Thomsen VØ, Johansen IS, et al. Nontuberculous pulmonary mycobacteriosis in Denmark: incidence and prognostic factors. Am J Respir Crit Care Med 2010; 181: 514-521.

187 Schwiesow JN, Iseman MD, Peloquin CA. Concomitant use of voriconazole and rifabutin in a patient with multiple infections. Pharmacotherapy 2008; 28: 1076-1080.

188 Johnston ID. Mycobacterium xenopi infection and aspergilloma. Tubercle 1988; 69: 139-143.

189 Carrillo MC, Patsios D, Wagnetz U, et al. Comparison of the spectrum of radiologic and clinical manifestations of pulmonary disease caused by Mycobacterium avium complex and Mycobacterium xenopi. Can Assoc Radiol J 2014; 65: 207-213.

190 Ferro BE, van Ingen J, Wattenberg M, et al. Time-kill kinetics of slowly growing mycobacteria common in pulmonary disease. J Antimicrob Chemother 2015; 70: 2838-2843.

191 Andréjak C, Almeida DV, Tyagi S, et al. Improving existing tools for Mycobacterium xenopi treatment: assessment of drug combinations and characterization of mouse models of infection and chemotherapy. $J$ Antimicrob Chemother 2013; 68: 659-665.

192 Lounis N, Truffot-Pernot C, Bentoucha A, et al. Efficacies of clarithromycin regimens against Mycobacterium xenopi in mice. Antimicrob Agents Chemother 2001; 45: 3229-3230.

193 Marusić A, Katalinić-Janković V, Popović-Grle S, et al. Mycobacterium xenopi pulmonary disease: epidemiology and clinical features in non-immunocompromised patients. J Infect 2009; 58: 108-112.

194 Tortoli E, Kohl TA, Brown-Elliott BA, et al. Emended description of Mycobacterium abscessus, Mycobacterium abscessus subsp. abscessus and Mycobacterium abscessus subsp. bolletii and designation of Mycobacterium abscessus subsp. massiliense comb. nov. Int J Syst Evol Microbiol 2016; 66: 4471-4479.

195 Pasipanodya JG, Ogbonna D, Ferro BE, et al. Systematic review and meta-analyses of the effect of chemotherapy on pulmonary Mycobacterium abscessus outcomes and disease recurrence. Antimicrob Agents Chemother 2017; 61: e01206-17.

196 Griffith DE, Girard WM, Wallace RJ Jr. Clinical features of pulmonary disease caused by rapidly growing mycobacteria: an analysis of 154 patients. Am Rev Respir Dis 1993; 147: 1271-1278.

197 van Ingen J, de Zwaan R, Dekhuijzen RP, et al. Clinical relevance of Mycobacterium chelonae-abscessus group isolation in 95 patients. $J$ Infect 2009; 59: 324-331.

198 Lyu J, Jang HJ, Song JW, et al. Outcomes in patients with Mycobacterium abscessus pulmonary disease treated with long-term injectable drugs. Respir Med 2011; 105: 781-787.

199 Harada T, Akiyama Y, Kurashima A, et al. Clinical and microbiological differences between Mycobacterium abscessus and Mycobacterium massiliense lung diseases. J Clin Microbiol 2012; 50: 3556-3561.

200 Tung YJ, Bittaye SO, Tsai JR, et al. Risk factors for microbiologic failure among Taiwanese adults with Mycobacterium abscessus complex pulmonary disease. J Microbiol Immunol Infect 2015; 48: 437-445.

201 Griffith DE, Philley JV, Brown-Elliott BA, et al. The significance of Mycobacterium abscessus subspecies abscessus isolation during Mycobacterium avium complex lung disease therapy. Chest 2015; 147: 1369-1375.

202 Namkoong H, Morimoto K, Nishimura T, et al. Clinical efficacy and safety of multidrug therapy including thrice weekly intravenous amikacin administration for Mycobacterium abscessus pulmonary disease in outpatient settings: a case series. BMC Infect Dis 2016; 16: 396.

203 Park J, Cho J, Lee $\mathrm{CH}$, et al. Progression and treatment outcomes of lung disease caused by Mycobacterium abscessus and Mycobacterium massiliense. Clin Infect Dis 2017; 64: 301-308.

204 Koh WJ, Jeong BH, Jeon K, et al. Oral macrolide therapy following short-term combination antibiotic treatment of Mycobacterium massiliense lung disease. Chest 2016; 150: 1211-1221.

205 Ellender CM, Law DB, Thomson RM, et al. Safety of IV amikacin in the treatment of pulmonary non-tuberculous mycobacterial disease. Respirology 2016; 21: 357-362.

206 Roux AL, Catherinot E, Soismier N, et al. Comparing Mycobacterium massiliense and Mycobacterium abscessus lung infections in cystic fibrosis patients. J Cyst Fibros 2015; 14: 63-69.

207 Lyu J, Kim BJ, Kim BJ, et al. A shorter treatment duration may be sufficient for patients with Mycobacterium massiliense lung disease than with Mycobacterium abscessus lung disease. Respir Med 2014; 108: 1706-1712.

208 Choi GE, Shin SJ, Won CJ, et al. Macrolide treatment for Mycobacterium abscessus and Mycobacterium massiliense infection and inducible resistance. Am J Respir Crit Care Med 2012; 186: 917-925.

209 Wang D, Fu W, Dai J. Meta-analysis of macrolide maintenance therapy for prevention of disease exacerbations in patients with noncystic fibrosis bronchiectasis. Medicine (Baltimore) 2019; 98: e15285.

210 Kelly C, Chalmers JD, Crossingham I, et al. Macrolide antibiotics for bronchiectasis. Cochrane Database Syst Rev 2018; 3: CD012406.

211 Aksamit TR, O'Donnell AE, Barker A, et al. Adult patients with bronchiectasis: a first look at the US Bronchiectasis Research Registry. Chest 2017; 151: 982-992.

212 Novosad SA, Beekmann SE, Polgreen PM, et al. Treatment of Mycobacterium abscessus infection. Emerg Infect Dis 2016; 22: 511-514.

213 Wang CC, Lin MC, Liu JW, et al. Nontuberculous mycobacterial lung disease in southern Taiwan. Chang Gung Med J 2009; 32: 499-508. 
214 Nelson KG, Griffith DE, Brown BA, et al. Results of operation in Mycobacterium avium-intracellulare lung disease. Ann Thorac Surg 1998; 66: 325-330.

215 Koh WJ, Kim YH, Kwon OJ, et al. Surgical treatment of pulmonary diseases due to nontuberculous mycobacteria. J Korean Med Sci 2008; 23: 397-401.

216 Yu JA, Pomerantz M, Bishop A, et al. Lady Windermere revisited: treatment with thoracoscopic lobectomy/ segmentectomy for right middle lobe and lingular bronchiectasis associated with non-tuberculous mycobacterial disease. Eur J Cardiothorac Surg 2011; 40: 671-675.

217 Kang HK, Park HY, Kim D, et al. Treatment outcomes of adjuvant resectional surgery for nontuberculous mycobacterial lung disease. BMC Infect Dis 2015; 15: 76.

218 Shiraishi Y, Fukushima K, Komatsu H, et al. Early pulmonary resection for localized Mycobacterium avium complex disease. Ann Thorac Surg 1998; 66: 183-186.

219 Shiraishi Y, Nakajima Y, Katsuragi N, et al. Pneumonectomy for nontuberculous mycobacterial infections. Ann Thorac Surg 2004; 78: 399-403.

220 Shiraishi Y, Nakajima Y, Takasuna K, et al. Surgery for Mycobacterium avium complex lung disease in the clarithromycin era. Eur J Cardiothorac Surg 2002; 21: 314-318.

221 Lang-Lazdunski L, Offredo C, Le Pimpec-Barthes F, et al. Pulmonary resection for Mycobacterium xenopi pulmonary infection. Ann Thorac Surg 2001; 72: 1877-1882.

222 Watanabe M, Hasegawa N, Ishizaka A, et al. Early pulmonary resection for Mycobacterium avium complex lung disease treated with macrolides and quinolones. Ann Thorac Surg 2006; 81: 2026-2030.

223 van Ingen J, Verhagen AF, Dekhuijzen PN, et al. Surgical treatment of nontuberculous mycobacterial lung disease: strike in time. Int J Tuberc Lung Dis 2010; 14: 99-105.

224 Koh WJ, Jeong BH, Jeon K, et al. Therapeutic drug monitoring in the treatment of Mycobacterium avium complex lung disease. Am J Respir Crit Care Med 2012; 186: 797-802.

225 Magis-Escurra C, Alffenaar JW, Hoefnagels I, et al. Pharmacokinetic studies in patients with nontuberculous mycobacterial lung infections. Int J Antimicrob Agents 2013; 42: 256-261.

226 Jeong BH, Jeon K, Park HY, et al. Peak plasma concentration of azithromycin and treatment responses in Mycobacterium avium complex lung disease. Antimicrob Agents Chemother 2016; 60: 6076-6083.

227 Nahid P, Dorman SE, Alipanah N, et al. Official American Thoracic Society/ Centers for Disease Control and Prevention/Infectious Diseases Society of America Clinical Practice Guidelines: treatment of drug-susceptible tuberculosis. Clin Infect Dis 2016; 63: e147-e195.

228 Daniel-Wayman S, Abate G, Barber DL, et al. Advancing translational science for pulmonary NTM infections: a roadmap for research. Am J Respir Crit Care Med 2019; 99: 947-951.

229 Henkle E, Aksamit T, Barker A, et al. Patient-centered research priorities for pulmonary nontuberculous mycobacteria (NTM) infection. An NTM Research Consortium Workshop Report. Ann Am Thorac Soc 2016; 13: S379-S384. 